Striped pattern selection by advective reaction-diffusion systems: Resilience of banded vegetation on slopes

E. Siero' , A. Doelman, M. B. Eppinga, J. D. M. Rademacher, M. Rietkerk, and K. Siteur

Citation: Chaos 25, 036411 (2015); doi: 10.1063/1.4914450

View online: http://dx.doi.org/10.1063/1.4914450

View Table of Contents: http://aip.scitation.org/toc/cha/25/3

Published by the American Institute of Physics

Welcome to a

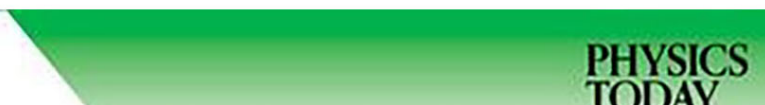

TODAY

with the redesigned

Physics Today Buyer's Guide

Find the tools you're looking for today! 


\title{
Striped pattern selection by advective reaction-diffusion systems: Resilience of banded vegetation on slopes
}

\author{
E. Siero, ${ }^{1, a)}$ A. Doelman, ${ }^{1}$ M. B. Eppinga, ${ }^{2}$ J. D. M. Rademacher, ${ }^{3}$ M. Rietkerk, ${ }^{2}$ \\ and K. Siteur ${ }^{2}$ \\ ${ }^{1}$ Mathematisch Instituut, Universiteit Leiden, P.O. Box 9512, 2300 RA Leiden, The Netherlands \\ ${ }^{2}$ Department of Environmental Sciences, Copernicus Institute, Faculty of Geosciences, Utrecht University, \\ P.O. Box 80115, 3508 TC, Utrecht, The Netherlands \\ ${ }^{3}$ Fachbereich Mathematik, Universität Bremen, Postfach 3304 40, 28359 Bremen, Germany
}

(Received 28 November 2014; accepted 27 February 2015; published online 17 March 2015)

\begin{abstract}
For water-limited arid ecosystems, where water distribution and infiltration play a vital role, various models have been set up to explain vegetation patterning. On sloped terrains, vegetation aligned in bands has been observed ubiquitously. In this paper, we consider the appearance, stability, and bifurcations of 2D striped or banded patterns in an arid ecosystem model. We numerically show that the resilience of the vegetation bands is larger on steeper slopes by computing the stability regions (Busse balloons) of striped patterns with respect to 1D and transverse 2D perturbations. This is corroborated by numerical simulations with a slowly decreasing water input parameter. Here, long wavelength striped patterns are unstable against transverse perturbations, which we also rigorously prove on flat ground through an Evans function approach. In addition, we prove a "Squire theorem" for a class of two-component reaction-advection-diffusion systems that includes our model, showing that the onset of pattern formation in 2D is due to 1D instabilities in the direction of advection, which naturally leads to striped patterns. (C) 2015 AIP Publishing LLC.

[http://dx.doi.org/10.1063/1.4914450]
\end{abstract}

This paper has been motivated by studies in one space dimension of a scaled phenomenological model for vegetation on possibly sloped planes in arid ecosystems. ${ }^{\mathbf{5 3 , 6 0}}$ One-dimensional patterns ideally represent striped patterns in two space dimensions by trivially extending them into a transversal direction. Such patterns are referred to as banded vegetation and have received considerable attention after reports of widespread observations. 8,58 Understanding the appearance and disappearance of vegetation bands may ultimately help prevent land degradation. The restriction to one space dimension may overestimate stability: patterns that are stable against 1D perturbations are not necessarily stable against all 2D perturbations. Natural questions to pose are:

- Which of the 1D stable patterns extend to 2D stable striped patterns?

- In case of destabilization by $2 \mathrm{D}$ perturbations, which mechanisms are responsible?

In this paper, we answer these questions for the arid ecosystem model and determine the impact of slope induced advection of water. The influence of advection on striped pattern formation is studied in a more general setting. This approach provides a clear argumentation that is unobscured by model-specific details. Equally important, the results will be applicable to a wide range of models. Applicability to the arid ecosystem model is carefully checked though, assuring that the abstract requirements can in fact be met in practice.

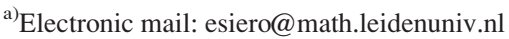

\section{INTRODUCTION}

The original Klausmeier model ${ }^{31}$ is an ecohydrological model for modeling vegetation patterns on sloped terrain in arid ecosystems, with a (surface) water component $w$ and a plant biomass or vegetation component $n$. The flow of water is modeled by downhill advection only. By adding a water diffusion term to the model, we arrive at the extended Klausmeier model ${ }^{53}$ studied in this article. In dimensionless form, it is given by

$$
\begin{aligned}
& w_{t}=d \Delta w+2 c w_{x}+a-w-w n^{2}, \\
& n_{t}=\Delta n-m n+w n^{2},
\end{aligned}
$$

where $\Delta=\frac{\partial^{2}}{\partial x^{2}}+\frac{\partial^{2}}{\partial y^{2}}$, posed on the plane. Generally the diffusion coefficient $d \gg 1$, since water diffuses much faster than vegetation. The parameter $c$ measures the advection of water down a hillslope, $a \geq 0$ models precipitation and $m>0$ an effective death rate. The terms $\pm w n^{2}$ model water uptake by vegetation and $-w$ evaporation.

The model (1) with $c=0$ and $a-w$ replaced by the term $a(1-w)$ is called the Gray-Scott model. ${ }^{26}$ The generalized form of (1) with the term $a(1-w)$ has been referred to as the generalized Klausmeier-Gray-Scott model; here also, the impact of nonlinear diffusion of the water component has been studied. ${ }^{39,60}$

Both the Klausmeier model and the Gray-Scott model exhibit patterns. ${ }^{31,40} \mathrm{We}$ will study the influence of the advection parameter $c$ on striped patterns. For fixed $c$, we view the rainfall parameter $a$ as the primary parameter to vary, as done in previous studies. ${ }^{53,60}$ 
The choice of parameter values in (1) will correspond to choices in Ref. 53, which are themselves based on Ref. 31. Since we are considering a scaled model, some of the parameters are a mix of parameters from the original Klausmeier model. ${ }^{31}$ For instance, what we refer to as slope driven advection $c$ in (1) is influenced by the evaporation rate from the original model, which has been scaled to 1 in (1). ${ }^{53}$ So, determining which values of $c$ are realistic is non-trivial. This is resolved by choosing $c$ over a wide range, giving an overview of the different possibilities. Unless stated otherwise, $m=0.45$ and $d=500$.

The Busse balloon ${ }^{4}$ is a representation of spatially periodic stable patterns that exist in a system; each pattern is represented by its wavenumber $\kappa:=2 \pi /$ wavelength. Uniting the stable patterns for a range of parameter values creates a planar region. For (1) on flat ground (no advection, $c=0$ ), the Busse balloon of 1D stable patterns is illustrated by the union of the two colored regions in Figure $1 .{ }^{53}$ The extensions of these $1 \mathrm{D}$ patterns to $2 \mathrm{D}$ striped patterns, which are represented by the same single wavenumber, are only $2 \mathrm{D}$ stable in the dark-green (teal) region near the Turing bifurcation $T$. In Sec. III C, the nature and construction of these two types of Busse balloons will be considered. Moreover, we will study the influence of slope induced advection of water $c$ and compare the stability results with simulations with a slowly decreasing rainfall parameter $a$.

In $1 \mathrm{D}$, in simulations with decreasing rainfall $a$, the dynamics (after pattern formation) is essentially restricted to transitions from one pattern to another, before reaching the bare desert state. ${ }^{53}$ In $2 \mathrm{D}$, these transitions correspond to stripe-to-stripe pattern transitions. Regarding striped patterns in $2 \mathrm{D}$, the additional instabilities we find always induce an amplitude modulation in the transverse direction along the stripes so that the bifurcating solutions decompose into spots, in analogy to findings focussing on a single (homoclinic) stripe. ${ }^{19,32,35}$ We find that destabilizing modes relate neighboring stripes either synchronously, leading to a stripe-torectangle pattern transition, or phase shifted by half a period, leading to a stripe-to-rhomb pattern transition. For this

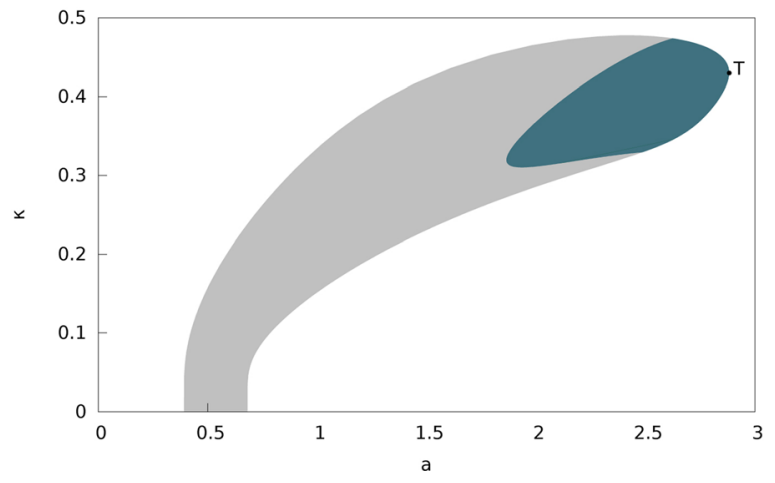

FIG. 1. Busse balloon representation of striped patterns on flat ground $(c=0)$ for the extended Klausmeier model (1) (with $d=500, m=0.45$ ). Here, each pattern is represented by a point in $(a, \kappa)$-space, where $\kappa$ $=2 \pi /$ wavelength is the wavenumber. A Turing bifurcation occurs at $T$. The union of the two colored regions consists of one-dimensional (spatially periodic) patterns that are $1 \mathrm{D}$ stable. ${ }^{53}$ The dark-green (teal) colored region consists of those patterns that extend to $2 \mathrm{D}$ stable striped patterns, patterns in grey extend to $2 \mathrm{D}$ unstable patterns. reason, and as a convenient terminology, we refer to these as (transverse) stripe-rectangle and stripe-rhomb breakup, respectively. Details are given in Sec. III A, where we will moreover trace the 2D patterns that bifurcate from the striped patterns numerically. ${ }^{20,57}$

The main numerical result for (1), framed in the terminology that is appropriate in this context, concerns the influence of the slope (advection, $c$ ) on banded (or striped) vegetation resilience. Ecological resilience is measured by the magnitude of disturbance that can be absorbed before the system redefines its structure. ${ }^{27,28}$ For (1), it holds that

(1) Increased resilience: the ecological resilience of banded vegetation is larger on steep slopes than on gentle slopes (Sec. III C).

Figure 1 shows numerically that for $c=0$ and small wavenumbers $\kappa$, none of the 1D patterns extend to 2D stable striped patterns. In accordance, we prove through the use of geometric singular perturbation theory and an Evans function approach that

(2) Transverse instability: in absence of advection (no slope, $c=0$ ) long wavelength striped patterns of (1) are unstable with respect to (w.r.t.) transverse instabilities (Corollary 2, Sec. III B).

Next to striped pattern (in)stability, it is also relevant to study the onset of striped pattern formation, and the influence of the advection $c$. The analytical results of Secs. II A and IIB are obtained in the setting of general twocomponent reaction-advection-diffusion systems. Specifically, we study systems posed on the plane

$$
\begin{gathered}
u_{t}=d_{1} \Delta u+c_{1} u_{x}+f(p, u, v), \\
v_{t}=d_{2} \Delta v+c_{2} v_{x}+g(p, u, v),
\end{gathered}
$$

where $p$ is an abstract parameter and $u(t, x, y), v(t, x, y) \in \mathbb{R}$. The advection coefficients $c_{1}, c_{2} \in \mathbb{R}$ are arbitrary, and we assume that $d_{1} \geq 0$ and $d_{2}>0$; compare Lemma 4 (Appendix A). We refer to the difference between the coefficients of the advection terms, $\left|c_{1}-c_{2}\right|$, as differential flow. ${ }^{45}$

We consider the linear stability of a homogeneous steady state $\left(u_{*}, v_{*}\right)$ of inhibitor-activator type that is stable against homogeneous perturbations. We define

$$
\begin{aligned}
a_{1} & :=\frac{\partial f}{\partial u}\left(u_{*}, v_{*}\right), \quad a_{2}:=\frac{\partial f}{\partial v}\left(u_{*}, v_{*}\right), \\
a_{3} & :=\frac{\partial g}{\partial u}\left(u_{*}, v_{*}\right), \quad a_{4}=\frac{\partial g}{\partial v}\left(u_{*}, v_{*}\right)
\end{aligned}
$$

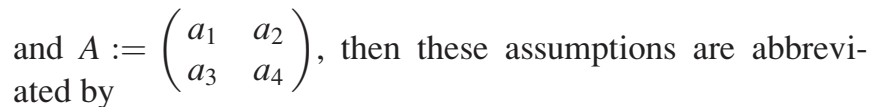

$$
\begin{aligned}
& A 1: \operatorname{tr}(A)<0 \text { and } \operatorname{det}(A)>0, \\
& A 2: a_{1}<0 \text { and } a_{4}>0,
\end{aligned}
$$

the latter meaning that $u$ acts as inhibitor (or depleted substrate) and $v$ acts as activator.

In Sec. II, we perform a thorough linear stability analysis near criticality. In case of no differential flow, $c_{1}=c_{2}$, destabilization of the homogeneous steady state occurs through a Turing instability leading to the emergence of 
stationary periodic patterns. In case of nonzero differential flow, $c_{1} \neq c_{2}$, the instability is referred to as Turing-Hopf (or oscillatory Turing) since the patterns that emerge are moving. The initial steps are as in Ref. 42, which starts out from the same setting. We derive the following novel results:

(3) Direction of motion: if $c_{1}>c_{2}$ and $c_{2} \leq 0$, then patterns emerging from the Turing-Hopf bifurcation move in the positive $x$-direction (Sec. II A 1).

(4) Locus monotonicity: the parameter location of the instability monotonically changes if the differential flow increases, assuming sign conditions on $\partial A / \partial p$ (Theorem 1, Sec. II A 2).

(5) Range monotonicity: the range of destabilizing perturbations of the homogeneous steady state monotically increases as the differential flow increases (Lemma 3, Sec. II B).

(6) Stripe formation dominance: for nonzero differential flow, perturbations independent of $y$ are responsible for the primary destabilization: at the Turing-Hopf bifurcation, striped patterns perpendicular to the direction of the advection appear (Sec. II B).

These general results are applicable to the arid ecosystem model (1), where the parameter $a$ takes on the role of the abstract parameter $p$. Interpreting the results in this context, we conclude that (within the model) small amplitude vegetation patterns move uphill. Second, under decreasing rainfall $a$ pattern formation first occurs on steeper slopes (Corollary 1, Sec. II C). As soon as the homogeneously vegetated state has become unstable against a specific perturbation, this will remain the case if the rainfall $a$ decreases more. And finally, banded vegetation perpendicular to the slope naturally forms on slopes. The paper ends with a discussion on ecological implications of the results regarding (1) and links to observations and comparison with other model studies in Sec. IV.

Remark 1. In Ref. 2, a listing of ecohydrological models with and without differential flow is given. The general results can be applied to various other disciplines, in particular, to differential flow models where the reactants have different advection coefficients. In chemical reactions between differently charged particle species, differential flow can be created by applying an exterior electric field. 5,37,56,61 Similarly, a differential flow induced chemical instability ("DIFICI") may be produced within a differential flow reactor with one particle species immobilized. ${ }^{38,45,46,49}$ Finally, also mussels that feed on algae, where (only) the algae flow with tidal currents, ${ }^{63}$ fit the abstract framework (2).

Remark 2. The present insights induce (novel) connections with fluid mechanics. The representation of stable patterns by Busse balloons originates from this field. ${ }^{4}$ Although fundamentally different, similar patterns exist, e.g., context striped patterns are commonly called roll-waves and the transverse instabilities of striped patterns we find correspond to certain "oblique-roll" instabilities. ${ }^{29}$ In both fields, the onset of pattern formation can be studied by weakly nonlinear stability theory, for instance, on pre-imposed lattices. $^{24,25}$ The transformation presented in the proof of Lemma 3 that lifts the $1 D$ results from Sec. II A to $2 D$, has a counterpart in fluid mechanics: the "Squire's transformation." It leads to the "Squire's theorem," 21,54 which is still an active topic of research ${ }^{30}$ Originally, it refers to the fact that for shear flow instability of the Orr-Sommerfeld equation, the critical Rayleigh number for a threedimensional parallel flow is determined by two-dimensional perturbations, which links to the restriction to y-independent perturbations in our striped pattern formation dominance result in Sec. II B.

\section{STRIPED PATTERN FORMATION}

We choose to first avoid model specific considerations and study pattern formation in the setting of the general system (2). We present a comprehensive linear analysis about a homogeneous steady state of inhibitor-activator type near onset of pattern formation. We start our analysis in one spatial dimension in Sec. II A but lift the results to two spatial dimensions in Sec. II B.

In Sec. II A 1, we establish the direction of motion of emerging patterns of (2) depending on the advection coefficients $c_{1}, c_{2}$. In Sec. II A 2, we prove a result on the monotonic change of the parameter locus of the Turing-Hopf instability as the differential flow $2 c=\left|c_{1}-c_{2}\right|$ increases, Theorem 1. One of the results leading up to this monotonicity result, Lemma 1 on the destabilizing impact of increasing $c$, plays an important role in carrying over the results from $1 \mathrm{D}$ to $2 \mathrm{D}$.

In Sec. II B, we show that for $c>0$, destabilization in $2 \mathrm{D}$ can be reduced to destabilization in $1 \mathrm{D}$ in the advection direction. We prove that the destabilization locus in 2D coincides with the locus in 1D, and we show that the set of destabilizing perturbations is strictly monotonically increasing with the advection $c$. It is shown that striped patterns naturally arise from a Turing-Hopf instability in 2D.

We subsequently apply the general insights to the extended Klausmeier model (1) in Sec. IIC. The abstract results in this context imply that on slopes, vegetation bands form that migrate uphill.

\section{A. Linear analysis of pattern formation for the general system in 1D}

The reduction of (2) to one dimension in the direction of the advection is

$$
\begin{aligned}
& u_{t}=d_{1} u_{x x}+c_{1} u_{x}+f(p, u, v), \\
& v_{t}=d_{2} v_{x x}+c_{2} v_{x}+g(p, u, v) .
\end{aligned}
$$

The linear stability of a homogeneous steady state of (5) can be determined by computing the linearization. Subsequently applying a Fourier transform yields the matrix

$$
M=\left(\begin{array}{cc}
-d_{1} k^{2}+\mathrm{i} c_{1} k+a_{1} & a_{2} \\
a_{3} & -d_{2} k^{2}+\mathrm{i} c_{2} k+a_{4}
\end{array}\right),
$$

where $k$ is the wavenumber of the perturbation and $a_{j}$ are the derivatives relevant for determining stability against homogeneous perturbations (see (3)). The linear dispersion relation is given by

$$
d(\lambda, k, p, c):=\operatorname{det}(M-\lambda I)=\lambda^{2}+\alpha_{1} \lambda_{1}+\alpha_{0}=0,
$$


where $\alpha_{1}=-\operatorname{tr}(M)$ and $\alpha_{0}=\operatorname{det}(M)$. The eigenvalues $\lambda$ of $M$, which are solutions to (6), determine (in)stability.

We are free to choose a suitable frame of reference, as the (in)stability of the homogeneous steady state does not depend on it. By changing the reference frame, we can manipulate the coefficients $\alpha_{1}$ and $\alpha_{0}$ in the dispersion relation. When changing to a moving reference frame with speed $\tilde{c}$, i.e., by the substitution $x \mapsto x-\tilde{c} t$, only the time derivative in (5) transforms, e.g., $u_{t} \mapsto u_{t}-\tilde{c} u_{x}$. When reflecting space $x \mapsto-x$, only the single derivatives to space are affected, e.g., $u_{x} \mapsto-u_{x}$. So the equations can be transformed into the equivalent

$$
\begin{aligned}
u_{t} & =d_{1} u_{x x} \pm\left(c_{1}+\tilde{c}\right) u_{x}+f(p, u, v), \\
v_{t} & =d_{2} v_{x x} \pm\left(c_{2}+\tilde{c}\right) v_{x}+g(p, u, v),
\end{aligned}
$$

where the negative sign applies in case of reflection.

A reference frame that will show to be suitable for stability analysis is obtained by choosing $\tilde{c}=-\left(c_{1}+c_{2}\right) / 2$, so that both advection coefficients have equal absolute value $c=\left|c_{1}-c_{2}\right| / 2$ but opposite sign. By means of spatial reflection, we can arrange that the first component (inhibitor) advection coefficient has positive sign. So we arrive at the form

$$
\begin{aligned}
& u_{t}=d_{1} \Delta u+c u_{x}+f(p, u, v), \\
& v_{t}=d_{2} \Delta v-c v_{x}+g(p, u, v),
\end{aligned}
$$

with a larger $c$ meaning a larger differential flow.

In this reference, frame $\alpha_{1}$ and $\alpha_{0}$ are given by

$$
\begin{aligned}
& \alpha_{1}=\left(d_{1}+d_{2}\right) k^{2}-a_{1}-a_{4}, \\
& \alpha_{0}=\left(-d_{1} k^{2}+\mathrm{i} c k+a_{1}\right)\left(-d_{2} k^{2}-\mathrm{i} c k+a_{4}\right)-a_{2} a_{3} .
\end{aligned}
$$

Under assumption $A 1, \operatorname{tr}(A)<0$, which implies $\alpha_{1}>0$. The real and imaginary parts of $\alpha_{0}$ are given by

$$
\begin{aligned}
\operatorname{Re}\left(\alpha_{0}\right) & =\left(-d_{1} k^{2}+a_{1}\right)\left(-d_{2} k^{2}+a_{4}\right)+c^{2} k^{2}-a_{2} a_{3}, \\
& =d_{1} d_{2} k^{4}-\Gamma k^{2}+c^{2} k^{2}+\operatorname{det}(A), \\
\operatorname{Im}\left(\alpha_{0}\right) & =c k\left(\left(d_{1}-d_{2}\right) k^{2}+a_{4}-a_{1}\right),
\end{aligned}
$$

where we introduced $\Gamma:=d_{1} a_{4}+d_{2} a_{1}$.

For the purpose of self-containment, we briefly treat the well-known Turing instability $(c=0)$ first. In this case, the dispersion relation $(6)$ reduces to

$$
\lambda^{2}+\left(\left(d_{1}+d_{2}\right) k^{2}-\operatorname{tr}(A)\right) \lambda+d_{1} d_{2} k^{4}-\Gamma k^{2}+\operatorname{det}(A)=0,
$$

and implicit differentiation with respect to $k$ yields

$$
\begin{aligned}
& 2 \lambda\left(\frac{\partial \lambda}{\partial k}+\left(d_{1}+d_{2}\right) k\right)+\left(\left(d_{1}+d_{2}\right) k^{2}-\operatorname{tr}(A)\right) \frac{\partial \lambda}{\partial k} \\
& \quad+4 d_{1} d_{2} k^{3}-2 \Gamma k=0 .
\end{aligned}
$$

Imposing stationary criticality $(\lambda=0)$ and that the spectrum is tangential $\left(\frac{\partial \lambda}{\partial k}=0\right)$ gives

$$
d_{1} d_{2} k^{4}-\Gamma k^{2}+\operatorname{det}(A)=0
$$

$$
4 d_{1} d_{2} k^{3}-2 \Gamma k=0 .
$$

It follows from the last equation that $\Gamma=2 d_{1} d_{2} k^{2}>0$ and insertion into the former equation gives $\Gamma^{2}$ $=4 d_{1} d_{2} \operatorname{det}(A)$, thus a Turing instability occurs if and only if

$$
\Gamma=2 \sqrt{d_{1} d_{2} \operatorname{det}(A)} .
$$

Concerning general $c$, we now develop some useful reference material for critical cases where $\operatorname{Re}(\lambda)=0$. Here, the dispersion relation (6) reduces to an expression that will prove to be insightful.

Isolating the imaginary part of (6) and imposing $\operatorname{Re}(\lambda)=0$ yields

$$
\operatorname{Re}\left(\alpha_{1}\right) \operatorname{Im}(\lambda)+\operatorname{Im}\left(\alpha_{0}\right)=0 .
$$

In the reference frame of (8), it holds that $\operatorname{Im}\left(\alpha_{1}\right)=0$, so that $\operatorname{Im}(\lambda)=-\frac{\operatorname{Im}\left(\alpha_{0}\right)}{\alpha_{1}}$. If we now combine this with the real part of (6), we obtain

$$
\alpha_{1}^{2} \operatorname{Re}\left(\alpha_{0}\right)-\operatorname{Im}\left(\alpha_{0}\right)^{2}=0 .
$$

Upon substituting equalities from (9) and (10) into (13) and some rewriting, we obtain the following polynomial equation in $k^{2}$ :

$$
\begin{aligned}
& {\left[\left(d_{1}+d_{2}\right) k^{2}-\operatorname{tr}(A)\right]^{2}\left[\left(d_{1} d_{2} k^{2}-\Gamma\right) k^{2}+\operatorname{det}(A)\right]} \\
& \quad+4 c^{2}\left[\left(d_{1} d_{2} k^{2}-\Gamma\right) k^{2}+a_{1} a_{4}\right] k^{2}=0
\end{aligned}
$$

First note that all terms in (14) are positive except $a_{1} a_{4}<0$ on the second line and possibly $d_{1} d_{2} k^{2}-\Gamma$, which appears on both lines. For $c=0$, this confirms the well-known fact that $\Gamma>0$ is a necessary condition for a Turing instability, see (11). On the other hand, this shows that the instability can also be purely driven by ramping up the advection $c$ since the only $c$-dependent term $4 c^{2}\left(\left(d_{1} d_{2} k^{2}-\Gamma\right) k^{2}+a_{1} a_{4}\right)$ is negative for $k^{2}$ small, relating to differential flow instabilities mentioned in the introduction.

We continue with some useful estimates that can be derived from (14). We first note an upper bound for the wavenumbers that can become critical given by

$$
k^{2}<\frac{a_{4}}{d_{2}} .
$$

Suppose on the contrary that $k^{2} \geq \frac{a_{4}}{d_{2}}$, then $d_{1} d_{2} k^{2}-\Gamma$ $\geq-d_{2} a_{1}>0$. Thus, also

$$
\left(d_{1} d_{2} k^{2}-\Gamma\right) k^{2}+a_{1} a_{4} \geq-d_{2} a_{1} k^{2}+a_{1} a_{4} \geq 0 .
$$

Now, all terms of (14) are positive for $k^{2} \in\left[\frac{a_{4}}{d_{2}}, \infty\right)$, so these wavenumbers cannot be critical.

Hence, there exists an upper bound on the wavenumber of destabilizing perturbations, independent of $c,{ }^{42}$ determined by the activator only. For future reference, we note that as a consequence of (15), it holds that 


$$
\begin{aligned}
\frac{\operatorname{Im}\left(\alpha_{0}\right)}{c k} \pm \alpha_{1} \geq \frac{\operatorname{Im}\left(\alpha_{0}\right)}{c k}-\alpha_{1}= & \left(d_{1}-d_{2}\right) k^{2}+a_{4}-a_{1} \\
& -\left(\left(d_{1}+d_{2}\right) k^{2}-a_{1}-a_{4}\right) \\
= & 2\left(a_{4}-d_{2} k^{2}\right)>0 .
\end{aligned}
$$

\section{Motion of emerging patterns}

Here, we determine the direction of motion of patterns emerging from a destabilized homogeneous state, for advection coefficients $c_{1}>c_{2}$ and $c_{2} \leq 0$, by applying the reference frame independent result (15). We first do this for the limiting case $c_{2}=0<c_{1}$, as in (1). In this reference frame, the coefficients of the dispersion relation (6) are

$$
\begin{aligned}
& \alpha_{1}^{\prime}=\left(d_{1}+d_{2}\right) k^{2}-a_{1}-a_{4}-\mathrm{i} c_{1} k, \\
& \alpha_{0}^{\prime}=\left(-d_{1} k^{2}+\mathrm{i} c_{1} k+a_{1}\right)\left(-d_{2} k^{2}+a_{4}\right)-a_{2} a_{3} .
\end{aligned}
$$

The real part of $\alpha_{1}^{\prime}$ and the imaginary part of $\alpha_{0}^{\prime}$ are given by

$$
\begin{aligned}
& \operatorname{Re}\left(\alpha_{1}^{\prime}\right)=\alpha_{1}>0, \\
& \operatorname{Im}\left(\alpha_{0}^{\prime}\right)=c_{1} k\left(-d_{2} k^{2}+a_{4}\right) .
\end{aligned}
$$

Now, the speed $s^{\prime}$ at onset is given by

$$
s^{\prime}=\frac{\operatorname{Im}(\lambda)}{k}=\frac{\operatorname{Im}\left(\alpha_{0}^{\prime}\right)}{k \operatorname{Re}\left(\alpha_{1}^{\prime}\right)}=\frac{c_{1}\left(-d_{2} k^{2}+a_{4}\right)}{\alpha_{1}}>0,
$$

by (12), (15), and since $c_{1}>0$. The positive speed means that the direction of movement at criticality is in the positive $x$-direction; we further note that the speed increases linearly with $c_{1}$.

Let $s$ denote the speed of emerging patterns in a system with $c_{2}<0$ but still $c_{2}<c_{1}$. This system can be brought into the form of the limiting case $c_{2}=0$ by substituting $x \mapsto x+c_{2} t$, so that $s=s^{\prime}-c_{2}>0$, since $s^{\prime}>0$ and $c_{2}<0$. So, movement is again in the positive $x$-direction. By reflection symmetry, it is clear that if $c_{1}<c_{2}$ and $c_{2} \geq 0$, then emerging patterns move in the negative $x$-direction.

Additionally, if we fix $c$ and $p$, we can determine the influence of an incremental change of the wavenumber $k$ on the speed at criticality. This influence is independent of the reference frame; we compute

$$
\frac{\partial s^{\prime}}{\partial k^{2}}=\frac{-2 c\left(d_{2} \alpha_{1}+\left(-d_{2} k^{2}+a_{4}\right)\left(d_{1}+d_{2}\right)\right)}{\alpha_{1}^{2}}<0,
$$

(see (9)) so that at criticality, an increase in $k$ leads to a decrease in the speed.

Both the positivity of the speed $s^{\prime}$ and the influence of the wavenumber $k$ are in accordance with what was found previously in the context of (1), where water advection is downslope but vegetation bands move uphill. ${ }^{53}$

\section{Destabilization by $c$ and monotonicity of the destabilization locus}

The following lemma shows that for critical eigenvalues, an increase of differential flow, $c$, will make the corresponding perturbation destabilizing. We recall the assumption (4) on stability against homogeneous perturbations $A 1$ of a homogeneous steady state of inhibitor-activator type $A 2$.

Lemma 1. Suppose that we have a solution to the dispersion relation (6) with $\operatorname{Re}(\lambda)=0, c>0, k \neq 0, A 1$ and A2 hold, then $\operatorname{sgn} \operatorname{Re}\left(\frac{\partial \lambda}{\partial c}\right)>0$.

Proof. We implicitly differentiate (6) to $c$ while keeping $k$ and $p$ fixed:

$$
2 \lambda \frac{\partial \lambda}{\partial c}+\frac{\partial \alpha_{1}}{\partial c} \lambda+\alpha_{1} \frac{\partial \lambda}{\partial c}+\frac{\partial \alpha_{0}}{\partial c}=0,
$$

which leads to

$$
\frac{\partial \lambda}{\partial c}=-\frac{\frac{\partial \alpha_{1}}{\partial c} \lambda+\frac{\partial \alpha_{0}}{\partial c}}{2 \lambda+\alpha_{1}}=-\frac{\overline{2 \lambda+\alpha_{1}}}{\left|2 \lambda+\alpha_{1}\right|^{2}} \frac{\partial \alpha_{0}}{\partial c},
$$

since by (9) $\alpha_{1}$ is independent of $c$ (the bar denotes complex conjugation). Now by (12)

$$
\begin{aligned}
\left|2 \lambda+\alpha_{1}\right|^{2} \operatorname{Re}\left(\frac{\partial \lambda}{\partial c}\right) & =-\alpha_{1} \operatorname{Re}\left(\frac{\partial \alpha_{0}}{\partial c}\right)+2 \frac{\operatorname{Im}\left(\alpha_{0}\right)}{\alpha_{1}} \operatorname{Im}\left(\frac{\partial \alpha_{0}}{\partial c}\right) \\
& =-2 \alpha_{1} c k^{2}+2 \frac{\operatorname{Im}\left(\alpha_{0}\right)^{2}}{\alpha_{1} c} .
\end{aligned}
$$

Thus, it follows that

$$
\begin{aligned}
& \frac{\alpha_{1}}{2 c k^{2}}\left|2 \lambda+\alpha_{1}\right|^{2} \operatorname{Re}\left(\frac{\partial \lambda}{\partial c}\right)=\frac{\operatorname{Im}\left(\alpha_{0}\right)^{2}}{c^{2} k^{2}}-\alpha_{1}^{2} \\
& =\left(\frac{\operatorname{Im}\left(\alpha_{0}\right)}{c k}+\alpha_{1}\right)\left(\frac{\operatorname{Im}\left(\alpha_{0}\right)}{c k}-\alpha_{1}\right)>0
\end{aligned}
$$

by (16). Since $\alpha_{1}, c>0$, it holds that $\operatorname{Re}\left(\frac{\partial \lambda}{\partial c}\right)>0$.

We now include the parameter dependence of (8) in our treatment and assume that the homogeneous steady state persists as a function of the parameter $p$. Thus, the linearization $A=\left(\begin{array}{ll}a_{1} & a_{2} \\ a_{3} & a_{4}\end{array}\right)$ also becomes a function of $p$. We will now show that given sign conditions on $\partial A / \partial p$, an increase of $p$ will be either stabilizing or destabilizing.

Lemma 2. Suppose that we have a solution to the dispersion relation (6) with $\operatorname{Re}(\lambda)=0, A 1$ and $A 2$ hold and

$$
\operatorname{sgn} \frac{\partial a_{1}}{\partial p}=\operatorname{sgn} \frac{\partial a_{4}}{\partial p}=\operatorname{sgn} \frac{\partial \Gamma}{\partial p}=\operatorname{sgn}\left(-\frac{\partial \operatorname{det}(A)}{\partial p}\right),
$$

then $\operatorname{Re}\left(\frac{\partial \lambda}{\partial p}\right)$ carries the same sign.

Proof. Now implicit differentiation of (6) to $p$ while keeping $k$ and $c$ fixed gives

$$
2 \lambda \frac{\partial \lambda}{\partial p}+\frac{\partial \alpha_{1}}{\partial p} \lambda+\alpha_{1} \frac{\partial \lambda}{\partial p}+\frac{\partial \alpha_{0}}{\partial p}=0,
$$

which leads to

$$
\frac{\partial \lambda}{\partial p}=-\frac{\frac{\partial \alpha_{1}}{\partial p} \lambda+\frac{\partial \alpha_{0}}{\partial p}}{2 \lambda+\alpha_{1}}=-\frac{\left(\frac{\partial \alpha_{1}}{\partial p} \lambda+\frac{\partial \alpha_{0}}{\partial p}\right) \overline{2 \lambda+\alpha_{1}}}{\left|2 \lambda+\alpha_{1}\right|^{2}}
$$


Since $0<\alpha_{1} \in \mathbb{R}$, it holds that

$$
\begin{aligned}
\left|2 \lambda+\alpha_{1}\right|^{2} \operatorname{Re}\left(\frac{\partial \lambda}{\partial p}\right) \\
=-2 \lambda^{2} \operatorname{Re}\left(\frac{\partial \alpha_{1}}{\partial p}\right)-\operatorname{Re}\left(\frac{\partial \alpha_{0}}{\partial p}\right) \alpha_{1}-2 \lambda \operatorname{Im}\left(\frac{\partial \alpha_{0}}{\partial p}\right) \\
=2\left(\frac{\operatorname{Im}\left(\alpha_{0}\right)}{\alpha_{1}}\right)^{2}\left(\frac{\partial a_{1}}{\partial p}+\frac{\partial a_{4}}{\partial p}\right)-\left(-\frac{\partial \Gamma}{\partial p} k^{2}+\frac{\partial \operatorname{det}(A)}{\partial p}\right) \alpha_{1} \\
-2 \frac{\operatorname{Im}\left(\alpha_{0}\right)}{\alpha_{1}} c k\left(\frac{\partial a_{4}}{\partial p}-\frac{\partial a_{1}}{\partial p}\right) .
\end{aligned}
$$

Thus, $\alpha_{1}^{2}\left|2 \lambda+\alpha_{1}\right|^{2} \operatorname{Re}\left(\frac{\partial \lambda}{\partial p}\right)$ equals

$$
\begin{aligned}
2 \operatorname{Im}\left(\alpha_{0}\right)^{2}\left(\frac{\partial a_{1}}{\partial p}+\frac{\partial a_{4}}{\partial p}\right)-\left(-\frac{\partial \Gamma}{\partial p} k^{2}+\frac{\partial \operatorname{det}(A)}{\partial p}\right) \alpha_{1}^{3} \\
+2 c k \alpha_{1} \operatorname{Im}\left(\alpha_{0}\right)\left(\frac{\partial a_{1}}{\partial p}-\frac{\partial a_{4}}{\partial p}\right) \\
=c^{2} k^{2}\left(\frac{\operatorname{Im}\left(\alpha_{0}\right)}{c k}+\alpha_{1}+\frac{\operatorname{Im}\left(\alpha_{0}\right)}{c k}-\alpha_{1}\right) \\
\quad \times\left(\left(\frac{\operatorname{Im}\left(\alpha_{0}\right)}{c k}+\alpha_{1}\right) \frac{\partial a_{1}}{\partial p}+\left(\frac{\operatorname{Im}\left(\alpha_{0}\right)}{c k}-\alpha_{1}\right) \frac{\partial a_{4}}{\partial p}\right) \\
+\left(\frac{\partial \Gamma}{\partial p} k^{2}-\frac{\partial \operatorname{det}(A)}{\partial p}\right) \alpha_{1}^{3} .
\end{aligned}
$$

The factors in front of $\frac{\partial a_{1}}{\partial p}$ and $\frac{\partial a_{4}}{\partial p}$ are all positive by (16). Therefore, the signs of the terms in the final expression are determined by the signs in (20).

We now combine the results at criticality of Lemmas 1 and 2 together with some insights on the global influence of both the advection $c$ and the parameter $p$ on the stability of the homogeneous steady state. In the result below, "const" denotes a positive constant that may be different at any instance.

Theorem 1. We make a distinction between two cases.

Case 1. Let $I=\left[p_{1}, \infty\right)$ and assume that for $p \in I$ assumptions A1, A2 hold and Eq. (20) holds with sign -1. Assume that at $p=p_{1}$, the homogeneous steady state is linearly stable for some value of $c$. Then on I, the location of the instability $p_{T}$ is a strictly monotonically increasing function of $c$. If, moreover, there exists a $q \in I$ such that $p \geq q$ implies both $\Gamma(p) \leq 0$ and

$$
-2 \frac{\partial a_{1}}{\partial p}\left(\operatorname{det}(A)\left(d_{1}+d_{2}\right)-2 c^{2} a_{4}\right) \geq \mathrm{const},
$$

then $\lim _{c \rightarrow \infty} p_{T}=\infty$.

Case 2. Let $I=\left(0, p_{2}\right]$ and assume that for $p \in I$ assumptions A1, A2 hold and Eq. (20) holds with sign +1 . Assume that at $p=p_{2}$, the homogeneous steady state is linearly stable for some value of $c$. Then on I, the location of the instability $p_{T}$ is a strictly monotonically decreasing function of $c$. If, moreover, there exists a $q \in I$ such that $p \leq q$ implies both $\Gamma(p) \leq 0$ and

$$
-2 \frac{\partial a_{1}}{\partial p}\left(\operatorname{det}(A)\left(d_{1}+d_{2}\right)-2 c^{2} a_{4}\right) \leq \frac{- \text { const }}{p},
$$

then $\lim _{c \rightarrow \infty} p_{T}=0$.
Proof. Before making a case distinction, we do some preparatory work. As noted before, from expression (14), it can be seen that the stability of the homogeneous steady state can be manipulated by increasing $c$. Namely, there is only one term that depends on $c$, and for $k^{2}$ small, this term is approximated by $4 c^{2} a_{1} a_{4}<0$. By choosing the right value of $c$, it can be inferred that $\operatorname{Re}(\lambda)=0$ for some $k^{2}$ but nowhere $\operatorname{Re}(\lambda)>0$. Starting at this criticality, the following approximation can be made:

$$
\Delta \operatorname{Re}(\lambda) \approx \frac{\partial \operatorname{Re}(\lambda)}{\partial c} \Delta c+\frac{\partial \operatorname{Re}(\lambda)}{\partial p} \Delta p
$$

where $\Delta$ indicates an incremental change in the succeeding quantity. Thus, if we locally wish to trace criticality, then we should prescribe that

$$
\frac{\partial p}{\partial c}=-\frac{\frac{\partial \operatorname{Re}(\lambda)}{\partial c}}{\frac{\partial \operatorname{Re}(\lambda)}{\partial p}}
$$

How fast $p_{T}$ moves is now determined by the maximum/ minimum of (26) over all critical $\lambda$, a maximum/minimum that certainly exists since the dispersion relation is continuous and the evaluation is on a compact set $\left(k^{2}<\frac{a_{4}}{d_{2}}\right.$ by (15)).

On the other hand, we want to incorporate that a sufficient change in the parameter value may stabilize the homogeneous steady state. We pick the term $-2 \operatorname{tr}(A) \operatorname{det}(A)\left(d_{1}+d_{2}\right) k^{2}$ from (14) to counteract the negative term $4 c^{2} a_{1} a_{4} k^{2}$; thus, we are interested in the sign of

$$
4 c^{2} a_{1} a_{4}-2 \operatorname{tr}(A) \operatorname{det}(A)\left(d_{1}+d_{2}\right) .
$$

As a final preparatory step, we compute its derivative

$$
\begin{aligned}
\frac{\partial}{\partial p}( & \left.4 c^{2} a_{1} a_{4}-2 \operatorname{tr}(A) \operatorname{det}(A)\left(d_{1}+d_{2}\right)\right) \\
= & -2 \frac{\partial a_{1}}{\partial p}\left(\operatorname{det}(A)\left(d_{1}+d_{2}\right)-2 c^{2} a_{4}\right) \\
& +4 c^{2} a_{1} \frac{\partial a_{4}}{\partial p}-2 \frac{\partial a_{4}}{\partial p} \operatorname{det}(A)\left(d_{1}+d_{2}\right) \\
& -2 \operatorname{tr}(A) \frac{\partial \operatorname{det}(A)}{\partial p}\left(d_{1}+d_{2}\right) .
\end{aligned}
$$

We start making a distinction between the two cases.

Case 1. From Lemmas 1 and 2 and Eq. (26), it follows that $p_{T}$ is a monotonically increasing function. The only thing left to prove is that $\lim _{c \rightarrow \infty} p_{T}$ is unbounded. For this, it is sufficient to show that for any fixed $c$, the homogeneous steady state can always be stabilized by a sufficient increase of $p$. By choosing $p \geq q$, the only negative coefficient in (14) is $c^{2} a_{1} a_{4} k^{2}$. In this case, the last three terms of (28) are positive so

$$
\begin{aligned}
& \frac{\partial}{\partial p}\left(4 c^{2} a_{1} a_{4}-2 \operatorname{tr}(A) \operatorname{det}(A)\left(d_{1}+d_{2}\right)\right) \\
& \quad \geq-2 \frac{\partial a_{1}}{\partial p}\left(\operatorname{det}(A)\left(d_{1}+d_{2}\right)-2 c^{2} a_{4}\right) \geq \mathrm{const},
\end{aligned}
$$


for $p \geq q$ by assumption. So for $p$ large enough, the sign of (27) will be positive and (14) will have no solutions. Together with Lemma 4 (Appendix A), this implies stability of the homogeneous steady state.

Case 2. From Lemmas 1 and 2 and Eq. (26), it follows that $p_{T}$ is a monotonically decreasing function. The only thing left to prove is that $\lim _{c \rightarrow \infty} p_{T}=0$. For this, it is sufficient to show that for any fixed $c$, the homogeneous steady state can always be stabilized by a sufficient decrease of $p$. By choosing $p \leq q$, the only negative coefficient in (14) is $c^{2} a_{1} a_{4} k^{2}$. In this case, the last three terms of (28) are negative, so

$$
\begin{aligned}
& \frac{\partial}{\partial p}\left(4 c^{2} a_{1} a_{4}-2 \operatorname{tr}(A) \operatorname{det}(A)\left(d_{1}+d_{2}\right)\right) \\
& \quad \leq-2 \frac{\partial a_{1}}{\partial p}\left(\operatorname{det}(A)\left(d_{1}+d_{2}\right)-2 c^{2} a_{4}\right) \leq \frac{-\mathrm{const}}{p},
\end{aligned}
$$

for $p \leq q$ by assumption. Because $\int_{0}^{p \prime} \frac{1}{p} d p$ diverges, for $p$ small enough, the sign of (27) will again be positive and Eq. (14) has no solutions, again implying stability by Lemma 4 (Appendix A).

Uniqueness of the destabilization locus on $I$ for fixed $p$ or fixed $c$ is an immediate consequence of the strict monotonicity. We further note that in Theorem 1, case 2 can be reduced to case 1 by taking the parameter $1 / p$, so a more concise version without case distinction is possible. We refrain from implementing this because the present treatise fits better with the application to (1) that comes next.

\section{B. Linear analysis of pattern formation for the general system in 2D}

In this section, we study the destabilization of a homogeneous steady state of the general system (2) in two space dimensions, under assumption $A 1$ that the homogeneous steady state is stable against homogeneous perturbations and $A 2$ that the steady state is of the type inhibitor-activator (4).

We use the same reference frame (8) with advection coefficients $c_{1}=c$ and $c_{2}=-c$ as employed in deriving Theorem 1 in Sec. II A 2, where $c$ is a measure of differential flow, but we recall that (in)stability does not depend on the reference frame and results hold for general $c_{1}, c_{2}$. The dispersion relation (6) introduced in Sec. II A, whose solutions determine (in)stability, in 2D depends on two wavenumbers. The wavenumber in the direction of advection $x$ is again denoted $k$, the additional wavenumber for the $y$-direction is denoted $\ell$. Here, the dispersion relation is given by

$$
d(\lambda, k, \ell, c):=\operatorname{det}(M-\lambda I)=0,
$$

with $M$ given by

$M=\left(\begin{array}{cc}-d_{1}\left(k^{2}+\ell^{2}\right)+\mathrm{i} c k+a_{1} & a_{2} \\ a_{3} & -d_{2}\left(k^{2}+\ell^{2}\right)-\mathrm{i} c k+a_{4}\end{array}\right)$.

We can conveniently connect the results developed in Sec. II A to the stability in 2D through the following lemma.
Lemma 3. In the presence of differential flow, $c>0$, the primary destabilization of a homogeneous steady state of (2) satisfying $A 1$ and $A 2$ at criticality occurs through perturbations with $\ell=0$. The range of wavenumber pairs $(k, \ell)$ corresponding to destabilizing perturbations is strictly monotonically increasing with $c$, but bounded above by

$$
k^{2}+\ell^{2}<\frac{a_{4}}{d_{2}}
$$

Proof. We start with the important equivalence

$$
\begin{aligned}
& (\lambda, k, \ell, c) \text { solves }(29) \\
& \quad \Longleftrightarrow\left(\lambda, \sqrt{k^{2}+\ell^{2}}, 0, \frac{k c}{\sqrt{k^{2}+\ell^{2}}}\right) \text { solves }(29),
\end{aligned}
$$

which follows directly from the equality of the matrices $M$, see (30). Now suppose that the homogeneous steady state is marginally stable for some value of $c$. By the identity (32), the instability with respect to a perturbation with $\ell \neq 0$ is the same as the instability with respect to a perturbation with $\ell=0$ and smaller $c$ since $k / \sqrt{k^{2}+\ell^{2}}<1$. Due to the destabilizing impact of $c$ at criticality (see Lemma 1), 1D perturbations with smaller $c$ have $\operatorname{Re}(\lambda)<0$. Hence, the primary destabilization occurs through perturbations with $\ell=0$.

Strict monotonicity for pairs $(k, 0)$ is already due to Lemma 1. Because (in)stability against $(k, \ell)$ is linked to (in)stability against $\left(\sqrt{k^{2}+\ell^{2}}, 0\right)$ through $(32)$, this automatically extends to monotonicity for all $(k, \ell)$. The bound (15) extends likewise to (31).

Note that for $c=0$, symmetry implies that instability in any direction occurs simultaneously. As mentioned in the introduction, the transformation in (32) is known in fluid mechanics as "Squire's transformation." For a supercritical Turing(-Hopf) bifurcation, (stable) small amplitude patterns emerge for parameter values just beyond the bifurcation. In the subcritical case, the small amplitude patterns exist for parameter values just before the bifurcation. Lemma 3 immediately leads to the following result.

Theorem 2. The 2D destabilization locus coincides with the $1 D$ destabilization locus, so the monotonicity result Theorem 1 also holds in 2D. In case of a supercritical Turing-Hopf bifurcation, the primary patterns to form are striped patterns perpendicular to the advection.

\section{Application to the extended Klausmeier model}

In this section, we check applicability of the general results to the extended Klausmeier model (1). The spatially homogeneous steady states of (1) are given by $w_{\text {bare }}=a, n_{\text {bare }}=0$, and

$$
\begin{gathered}
w_{ \pm}=\frac{2 m^{2}}{a \pm \sqrt{a^{2}-4 m^{2}}}, \\
n_{ \pm}=\frac{a \pm \sqrt{a^{2}-4 m^{2}}}{2 m},
\end{gathered}
$$

for $a \geq 2 m .^{53}$ Since $\left(w_{-}, n_{-}\right)$is unstable against spatially homogeneous perturbations, ${ }^{53}$ we focus on the other vegetated state $\left(w_{+}, n_{+}\right)$. 
We provide some more elementary facts about (1), details can be found in Ref. 53. The linearization about $\left(w_{+}, n_{+}\right)$is given by the Jacobian matrix

$$
A=\left(\begin{array}{ll}
a_{1} & a_{2} \\
a_{3} & a_{4}
\end{array}\right)=\left(\begin{array}{cc}
-1-n_{+}^{2} & -2 m \\
n_{+}^{2} & m
\end{array}\right) .
$$

Thus, clearly, $a_{1}<0$ and $a_{4}>0$, so $\left(w_{+}, n_{+}\right)$is of inhibitoractivator type (assumption $A 2$, see (4)). It holds that $\operatorname{det}(A)=m\left(n_{+}^{2}-1\right)>0$ for $a>2 m$, but $\operatorname{tr}(A)<0$ if and only if

$$
m \leq 2 \quad \text { or } \quad a>\frac{m^{2}}{\sqrt{m-1}} .
$$

For these choices of parameters, $\left(w_{+}, n_{+}\right)$is stable against homogeneous perturbations (assumption $A 1$, see (4)). So, for a nonzero slope $c>0$, the uphill motion of patterns considered in Sec. II A 1 indeed applies. Moreover, Lemma 3 holds, so destabilization occurs through perturbations that are constant in the $y$-direction perpendicular to the direction of advection. After destabilization, the set of destabilizing perturbations becomes larger and larger.

For (1), the bound (31) on destabilizing perturbations reduces to $k^{2}+\ell^{2}<m$. In this paper, we work with the estimate $m=0.45$ for grass, for trees $m=0.045$ holds, ${ }^{31}$ and the bound is more restrictive. If $k=0$, then $c$ does not play a role and destabilization in the $y$-direction thus occurs independent of $c$, at $a \approx 2.883$. We will refer to this point as the anchor point $T_{y}$. The results from Lemma 3 are illustrated in Figure 2.

Checking the supercriticality condition of Theorem 2 analytically requires the computation of Landau coefficients, which is beyond the scope of this paper. Supercriticality has been proven in an asymptotic scaling of (1) in one space dimension, ${ }^{60}$ and numerically, it is found that this holds in a broad range of parameter space. Through Theorem 2, for advection $c>0$, the Turing-Hopf bifurcation is a natural mechanism for the formation of striped or banded vegetation patterns. We will see this formation of banded vegetation in simulations in Sec. III C.
To apply the monotonicity result Theorem 1, we need to check some more conditions. In the corollary below, it will be shown that, when the parameter $a$ assumes the role of the abstract parameter $p$, the parameter locus of its destabilization $a_{T}$ is a strictly monotonically increasing function of $c$ and $\lim _{c \rightarrow \infty} a_{T}=\infty$. Although within the scope of this paper the parameters $c$ and $a$ are most important, the theory developed here is also utilized to show that (when $m$ assumes the role of $p$ ) $m_{T}$ is a monotonically decreasing function of $c$ and $\lim _{c \rightarrow \infty} m_{T}=0$.

In preparation, we make note of some rough estimates for $n_{+}$

$$
\begin{aligned}
& n_{+} \leq \frac{a}{m}, \quad \frac{\partial n_{+}}{\partial m} \leq \frac{\partial}{\partial m} \frac{a}{2 m}=-\frac{a}{m^{2}}, \quad \frac{\partial n_{+}^{2}}{\partial a} \geq \frac{\partial}{\partial a} \frac{a^{2}}{4 m^{2}}=\frac{a}{2 m^{2}} \\
& \frac{\partial n_{+}^{2}}{\partial m} \leq \frac{\partial}{\partial m} \frac{a^{2}}{4 m^{2}}=\frac{-a^{2}}{2 m^{3}} .
\end{aligned}
$$

We recall that (1) is not precisely of the form (8) but can be brought into this form by changing the frame of reference, as detailed at the start of Sec. II A, and we apply Theorem 1 as if we have done so.

Corollary 1. Assume that (34) holds for $a=p_{1}$ and $m=p_{2}$ and that here the homogeneous steady state $\left(w_{+}, n_{+}\right)$ is linearly stable for some value of $c$.

Then for a on $\left[p_{1}, \infty\right)$ (with $m=p_{2}$ ), the location of the instability $a_{T}$ is a strictly monotonically increasing function of c. Moreover, $\lim _{c \rightarrow \infty} a_{T}=\infty$.

For $m$ on $\left(0, p_{2}\right]$ (with $\left.a=p_{1}\right)$, the location of the instability $m_{T}$ is a strictly monotonically decreasing function of $c$. Moreover, $\lim _{c \rightarrow \infty} m_{T}=0$.

Proof. The assumptions (4) of stability against homogeneous perturbations $A 1$ and being of inhibitor-activator type $A 2$ must now be checked for an interval of parameter values. The shape of the set of points $(a, m)$ satisfying $A 1$ given by (34) implies that if we pick $a=p_{1}$ and $m=p_{2}$ for which ( $w_{+}$, $\left.n_{+}\right)$is stable against homogeneous perturbations, then this remains true if $a$ is increased or $m$ is decreased, see Figure 4. The inhibitor-activator assumption was already found to

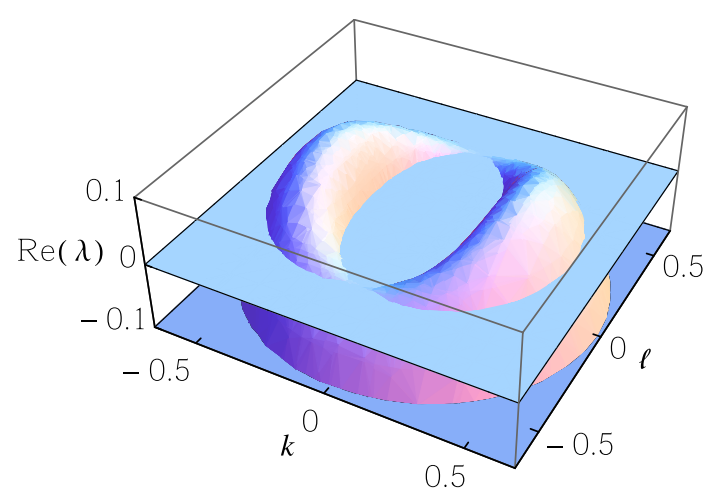

(a)

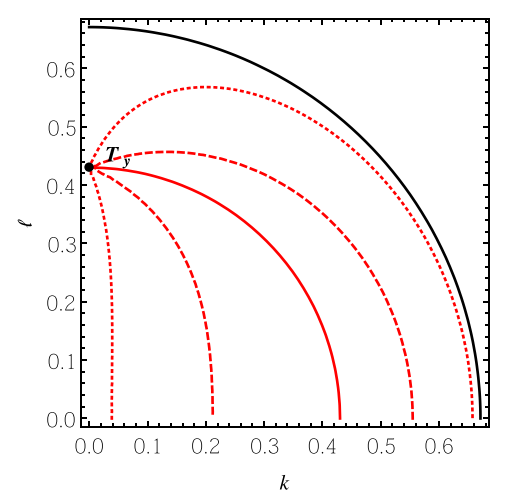

(b)

FIG. 2. The extended Klausmeier model (1) for $m=0.45(d=500)$ at $a \approx 2.883$ (onset of the Turing instability for $c=0$ ). (a) Real part of $\lambda(k, \ell, c)$ solving the homogeneous steady state dispersion relation (29) for $2 c=182.5$. Notably, $\operatorname{Re}(\lambda)$ is maximal for $\ell=0$. (b) Contour plots of $\operatorname{Re}(\lambda(k, \ell, c))=0$ for $c=0$ (red, continuous), $2 c=182.5$ (red, dashed), and $2 c=1000$ (red, dotted). For $c=0$, destabilization occurs in any direction simultaneously. For $c>0$, the range between the curves corresponding to destabilizing perturbations is increasing, but not beyond the black curve $k^{2}+\ell^{2}<m$. The anchor point $T_{y}$ appears at $k=0, \ell \approx 0.430$. 
hold everywhere. So, assumptions $A 1$ and $A 2$ hold for all $a \in\left[p_{1}, \infty\right)$ and $m \in\left(0, p_{2}\right]$.

For the parameter $a$, we apply (35) and readily compute that $\frac{\partial a_{1}}{\partial a}=-\frac{\partial n_{+}^{2}}{\partial a} \leq \frac{-a}{2 m^{2}} \leq \frac{-p_{1}}{2 m^{2}}, \frac{\partial a_{4}}{\partial a}=0, \frac{\partial \Gamma}{\partial a}=\frac{\partial a_{1}}{\partial a}$ and $\frac{\partial \operatorname{det}(A)}{\partial a}$ $=\frac{\partial a_{1}}{\partial a} m+2 m \frac{\partial a_{3}}{\partial a}=m \frac{\partial n_{+}^{2}}{\partial a} \geq \frac{a}{2 m} \geq \frac{p_{1}}{2 m}$. This shows that (20) holds with sign -1 , except that $\frac{\partial a_{4}}{\partial a}=0$, which is no problem as can be seen from the proof of Lemma 2. Since $\frac{\partial \Gamma}{\partial a} \leq \frac{-p_{1}}{2 m^{2}}$, $\Gamma$ is negative for $a$ large enough. With the help of the previous computations, we can make the following estimate to check (23):

$$
\begin{aligned}
& -2 \frac{\partial a_{1}}{\partial a}\left(\operatorname{det}(A)\left(d_{1}+d_{2}\right)-2 c^{2} a_{4}\right) \\
& \geq \frac{p_{1}}{m^{2}}\left(\left(\operatorname{det}\left(A\left(p_{1}\right)\right)+\left(a-p_{1}\right) \frac{p_{1}}{2 m}\right)\left(d_{1}+d_{2}\right)-2 c^{2} m\right),
\end{aligned}
$$

and the term on the right will become bigger then any constant if $a$ is taken large enough. So, case 1 of Theorem 1 applies to the parameter $a$.

For the parameter $m$, using (35), we compute that $\frac{\partial a_{1}}{\partial m}$ $=-\frac{\partial n_{+}^{2}}{\partial m} \geq \frac{a^{2}}{2 m^{3}}, \frac{\partial a_{4}}{\partial m}=1, \frac{\partial \Gamma}{\partial m}=d+\frac{\partial a_{1}}{\partial m}$ and

$$
\begin{aligned}
\frac{\partial \operatorname{det}(A)}{\partial m}= & \frac{\partial a_{1}}{\partial m} m+a_{1}+2 a_{3}+2 m \frac{\partial a_{3}}{\partial m} \\
= & m \frac{\partial n_{+}^{2}}{\partial m}-1+n_{+}^{2} \leq\left(2 m \frac{\partial n_{+}}{\partial m}+n_{+}\right) n_{+} \\
& \leq\left(2 m \frac{-a}{m^{2}}+\frac{a}{m}\right) \frac{a}{m}=\frac{-a}{m^{2}},
\end{aligned}
$$

so (20) holds with sign +1 . Clearly, $\Gamma=d m-1-n^{2}$ is negative for sufficiently small $\mathrm{m}$. By solving (36), we obtain

$$
\begin{aligned}
& -2 \frac{\partial a_{1}}{\partial m}\left(\operatorname{det}(A)\left(d_{1}+d_{2}\right)-2 c^{2} a_{4}\right) \\
& \leq-\frac{a^{2}}{m^{3}}\left(\left(\operatorname{det}\left(A\left(p_{2}\right)\right)-\frac{a}{2 p_{2}}+\frac{a}{2 m}\right)\left(d_{1}+d_{2}\right)-2 c^{2}\right) .
\end{aligned}
$$

The sign of the term on the right will become negative for $m$ small enough, also $\frac{1}{m^{3}}>\frac{1}{m}$ for $m<1$. Hence, case 2 of Theorem 1 applies to the parameter $m$.

Within the model (1), any choice of parameters that allows for a stable uniform vegetated state will behave as described by Corollary 1 . Thus, the locus of destabilization $a_{T}$ of the homogeneous steady state moves to higher $a$ as $c$ increases. This is consistent with what we find numerically. In Figure 3, we plot the values of $a_{T}$ for $2 c=0,182.5,365$, 500 , and 1000 , together with a square root function since $a_{T}$ grows as $\sqrt{c}$ for large $c$, in a certain scaling regime. ${ }^{60}$

Now that we understand the influence of both parameters $a$ and $m$, we can also fix $c$ and infer the dependence $a_{T}=a_{T}(m)$ for free. The following approximation complementary to (25) can be made:

$$
\Delta \operatorname{Re}(\lambda) \approx \frac{\partial \operatorname{Re}(\lambda)}{\partial a} \Delta a+\frac{\partial \operatorname{Re}(\lambda)}{\partial m} \Delta m,
$$

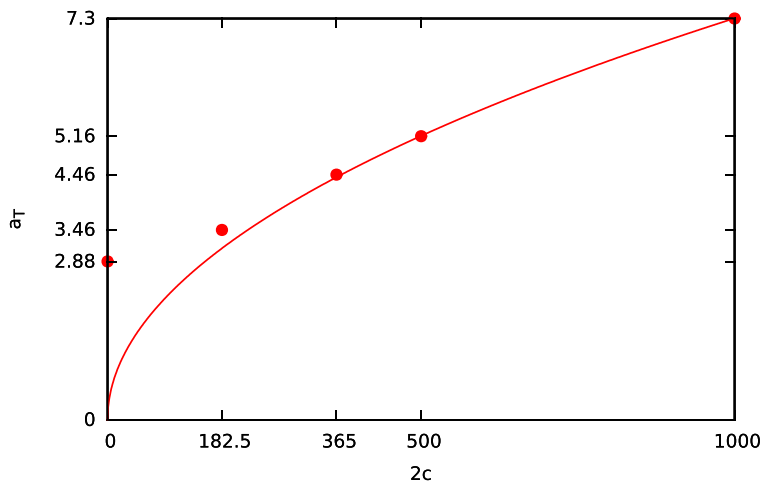

FIG. 3. The dots represent the location of the instability $a_{T}$ of (1) (with $d=500, m=0.45$ ) for several values of $2 c$. The result from Ref. 60 is illustrated by the graph of $0.231 \sqrt{2 c}$, with the factor chosen to fit the value at $2 c=1000$.

with $\Delta$ again indicating an incremental change. Now, if we locally wish to trace criticality, we should prescribe that

$$
\frac{\partial a}{\partial m}=-\frac{\frac{\partial \operatorname{Re}(\lambda)}{\partial m}}{\frac{\partial \operatorname{Re}(\lambda)}{\partial a}}>0,
$$

since we have already seen that $\frac{\partial \operatorname{Re}(\lambda)}{\partial m}>0$ and $\frac{\partial \operatorname{Re}(\lambda)}{\partial a}<0$ at criticality. To trace criticality $a$ and $m$ should be simultaneously increased or simultaneously decreased.

Figure 4 illustrates the different (in)stability regions of $\left(w_{+}, n_{+}\right)$for $c=0$. From Corollary 1, we know that, by increasing the advection $c$, a homogeneous steady state in the purple region $(d=500)$ can be made unstable. But, for any finite $c,\left(w_{+}, n_{+}\right)$is stable for $a$ large or $m$ small enough.

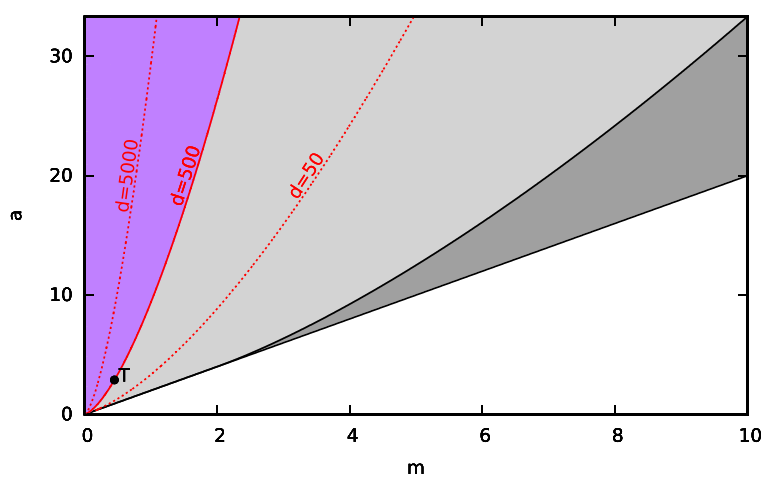

FIG. 4. Regions of stability of $\left(w_{+}, n_{+}\right)$for the extended Klausmeier model (1) for $c=0$. In the white region, $a<2 m$ and $\left(w_{+}, n_{+}\right)$do not exist. In the dark grey region where $2 m<a<\frac{m^{2}}{\sqrt{m-1}}, \operatorname{tr}(A)>0$. Elsewhere, $\left(w_{+}, n_{+}\right)$is stable against homogeneous perturbations. The red curves depict the solution set of $\Gamma=2 \sqrt{d \operatorname{det}(A)}$ for $d=50,500,5000$, see (11). At each of these curves $\left(w_{+}, n_{+}\right)$undergoes a Turing instability for the corresponding value of $d$. The relative placement of the curves as a function of $d$ is a consequence of $\Gamma / \sqrt{d}$ being an increasing function of $d$. By (37), $a_{T}=a_{T}(m)$ is a strictly monotonically increasing function of $m$, for every value of $d$. For $d=500$, in the light grey area, the homogeneous steady state is Turing unstable and in the purple area it is Turing stable. For $m=0.45$, the Turing instability occurs at $a \approx 2.883$, this point is labeled $T$ in the figure. 


\section{STRIPED PATTERN STABILITY IN 2D}

In this section, we restrict our attention to the extended Klausmeier model (1), where the differential flow equals the water advection.

Perturbations of the homogeneous state in 2D were represented by two wavenumbers $k, \ell \in \mathbb{R}$ for the $x$, $y$-directions, respectively, in Sec. IIB. In Sec. III A, we will first explain that perturbations of a pattern of stripes perpendicular to the direction of advection $x$ can be represented by $\ell \in \mathbb{R}$ and $\gamma$ on the unit circle $S^{1} \subset \mathbb{C}$, so that $\log (\gamma) \in(-\pi \mathrm{i}, \pi \mathrm{i}]$. The restriction to perturbations with $\ell=0$ corresponds to perturbations that were already considered in $1 \mathrm{D} .{ }^{53}$ Perturbations with $\ell \neq 0$ are not constant in the transverse $y$-direction along the stripes and may cause them to break up.

In Sec. III B, we analytically derive an instability result for localized striped patterns on flat ground ( $c=0$, no advection) through an Evans function approach, proving that in this case, a range of 1D stable patterns extends to 2D unstable striped patterns. These patterns will be unstable against perturbations for a range of values $\ell>0$, independent of $\gamma$.

We continue by numerically determining the collection of striped patterns that withstand the additional transverse destabilization mechanisms in Sec. III C. Here, perturbations with $\gamma= \pm 1$ play a special role, as stability against transverse perturbations for $\gamma= \pm 1$ seems to imply stability against all transverse perturbations. We show that the fraction of $2 \mathrm{D}$ stable striped patterns within the enveloping 1D Busse balloon increases as the advection $c$ increases, which is relevant for determining ecological resilience.

These results are complemented by simulations of (1) with the rainfall $a$ as a slowly decreasing parameter. As in Ref. 53, we trace the wavenumber of the solution if it is in a striped pattern state. The simulations show that the continuation method employed to determine striped pattern stability successfully predicts the occurrence of stripe breakup; depending on the advection $c$, the initial stages in the pattern selection process are determined by $1 \mathrm{D}$ effects $(\ell=0)$.

\section{A. Transverse instabilities: Breakup of stripes into rectangles or rhombs}

In Sec. II, we computed the linearization about a constant homogeneous state by representing perturbations by complex exponentials using the Fourier transform. Analogously, linearization about a periodic state with wavelength $2 \pi / \kappa$ is possible through a so-called Floquet-Bloch transform (see Appendix B). Now, perturbations are represented by functions $\tilde{n}_{\gamma}$ satisfying a " $\gamma$-twisted"3 periodicity property

$$
\tilde{n}_{\gamma}\left(x+\frac{2 \pi}{\kappa}\right)=\gamma \tilde{n}_{\gamma}(x),
$$

where $\gamma$ is on the unit circle (and similarly for the water component $w$ ). Note that $\gamma=1$ implies that the perturbation has exactly the same wavelength as the underlying pattern. The striped patterns we study are periodic in the $x$-direction and constant in the $y$-direction. Perturbations are thus represented by the combination $\tilde{n}(x, y)=n_{\gamma}(x) e^{\mathrm{i} \ell y}$.

As already noted, transverse perturbations with $\gamma= \pm 1$ turn out to be primary destabilization mechanisms, in Sec. III C. In this section, we will use numerical continuation in two spatial dimensions ${ }^{20,57}$ to visualize the destabilizing perturbations and the bifurcating 2D patterns for the cases $\gamma= \pm 1$, for the extended Klausmeier model (1). With these techniques, it becomes possible to map existence and stability of patterns periodic in $2 \mathrm{D}$, as we will illustrate, but an extensive search is outside the scope of this paper.

At the Turing-Hopf point where $a=a_{T}$, the homogeneous steady state is marginally stable against a perturbation with a distinct wavenumber $k_{T}$. Beyond the instability, a set of stable striped patterns exists (in the supercritical case), whose wavenumbers form an interval including $\kappa=k_{T}$. In Table I, we show at which value of $a_{\text {rect }}, a_{\text {rhomb }}$ this striped pattern becomes unstable against transverse breakup for $\gamma=1$, respectively, $\gamma=-1$ and at what distinct value of $\ell_{\text {rect }}, \ell_{\text {rhomb }}$ of the transverse wavenumber, for several values of $2 c$ (with $d=500$, $m=0.45$ ). The table is obtained by continuation methods for one spatial dimension, as will be used in Sec. III C.

Table I can be used as input to study striped pattern breakup, e.g., for $c=0$ in Figure 5. In order to find the 2D pattern that bifurcates from the striped pattern with $\kappa=k_{T}$ for $\gamma=1$, we start out from the homogeneous steady state $\left(w_{+}, n_{+}\right)$and choose $\left[0,4 \pi / k_{T}\right] \times\left[0,2 \pi / \ell_{\text {rect }}\right]$ as a domain. At the Turing(-Hopf) point, a two-stripe pattern (Figure 5(b)) bifurcates from $\left(w_{+}, n_{+}\right)$as the domain size in $x$ was prepared like this. If by continuation $a$ is decreased to $a_{\text {rect }}$, the two-stripe pattern becomes unstable against a transverse perturbation (Figure 5(c)) and a pattern periodic in both dimensions (Figure 5(d)) bifurcates. By (38), for $\gamma=1$, perturbations are in phase at neighboring stripes (at distance $2 \pi / \kappa)$ since $\tilde{n}\left(x+\frac{2 \pi}{\kappa}, y\right) \equiv \tilde{n}_{1}\left(x+\frac{2 \pi}{\kappa}\right) e^{\mathrm{i} \ell y}=\tilde{n}_{1}(x) e^{\mathrm{i} \ell y}=\tilde{n}(x, y)$. Periodically extending the pattern in Figure $5(\mathrm{~d})$ in both dimensions gives a rectangular pattern on the plane.

Likewise, for $\gamma=-1$, if we choose $\left[0,4 \pi / k_{T}\right]$ $\times\left[0,2 \pi / \ell_{\text {rhomb }}\right]$, the two-stripe pattern emerging at $a_{T}$ becomes unstable against a different transverse perturbation (Figure 6(c), where $2 c=182.5$ ) when $a$ is decreased to $a_{\text {rhomb }}$ and again a pattern periodic in both dimensions (Figure 6(d)) bifurcates. For $\gamma=-1$, perturbations are in opposite phase at neighboring stripes $(2 \pi / \kappa$ apart) since by (38) it holds that $\tilde{n}\left(x+\frac{2 \pi}{\kappa}, y\right) \equiv \tilde{n}_{-1}\left(x+\frac{2 \pi}{\kappa}\right) e^{\mathrm{i} \ell y}=$ $-\tilde{n}_{-1}(x) e^{\mathrm{i} \ell y} \equiv-\tilde{n}(x, y)$. Periodic extension of the pattern in

TABLE I. Table of Turing-Hopf loci $\left(a_{T}\right)$ of (1) (for $d=500, m=0.45$ ), the critical wavenumber at its onset $\left(k_{T}\right)$ and for the striped patterns with $\kappa=k_{T}$ the critical $a$-values and wavenumbers $\ell$ of perturbation along the striped pattern at breakup for $\gamma=1\left(a_{\text {rect }}\right.$ and $\left.\ell_{\text {rect }}\right)$ and $\gamma=-1\left(a_{\text {rhomb }}\right.$ and $\left.\ell_{\text {rhomb }}\right)$.

\begin{tabular}{lcccccc}
\hline \hline $2 c$ & $a_{T}$ & $k_{T}$ & $a_{\text {rect }}$ & $\ell_{\text {rect }}$ & $a_{\text {rhomb }}$ & $\ell_{\text {rhomb }}$ \\
\hline 0 & 2.883 & 0.430 & 2.232 & 0.433 & 2.297 & 0.410 \\
182.5 & 3.456 & 0.398 & 2.107 & 0.417 & 2.197 & 0.394 \\
365 & 4.460 & 0.384 & 2.011 & 0.418 & 2.197 & 0.365 \\
500 & 5.161 & 0.385 & 2.037 & 0.422 & 2.349 & 0.348 \\
1000 & 7.301 & 0.398 & 2.206 & 0.443 & 3.074 & 0.343 \\
\hline \hline
\end{tabular}



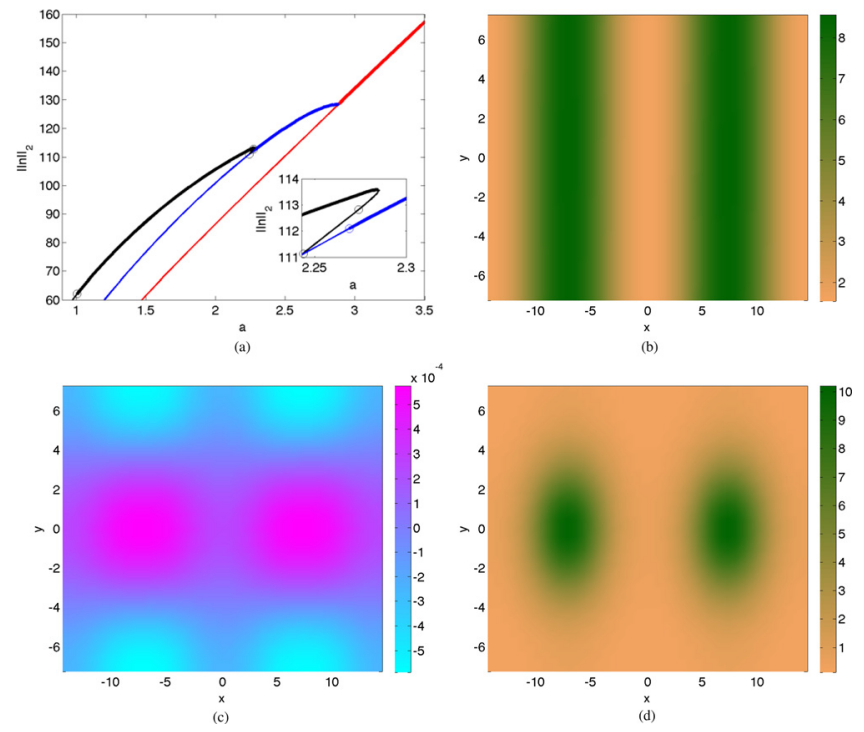

FIG. 5. (a) Stripe-rectangle bifurcation diagram of (1) for $c=0(d=500$, $m=0.45$ ) with branch of homogeneous equilibrium (red), bifurcating striped patterns with wavenumber $\kappa=k_{T}$ (blue), subcritically bifurcating rectangular pattern with $\ell=\ell_{\text {rect }}$ (black) and inset magnifying this subcritical bifurcation. For efficiency, the computations were done under zero flux Neumann boundary conditions, and thick lines indicate stability with respect to perturbations that fit the domain. Other panels show striped pattern (b) and destabilizing perturbation (c) at stripe-rectangle bifurcation point, rectangular pattern (d) at $a \approx 1$. Note that the solution plots extend periodically in both space directions.

Figure 6(d) yields a rhombic pattern. In this case, the bifurcating rhombic pattern deviates only little from a regular hexagonal pattern.

We note that the inset of Figure 5(a) shows that the branch of striped patterns becomes unstable before the stripe-rectangle bifurcation point is reached. This is because the stripe-rhomb bifurcation precedes the stripe-rectangle bifurcation, indeed $a_{\text {rhomb }}>a_{\text {rect }}$ and $\ell_{\text {rhomb }} \approx \ell_{\text {rect }}$ for $c=0$,
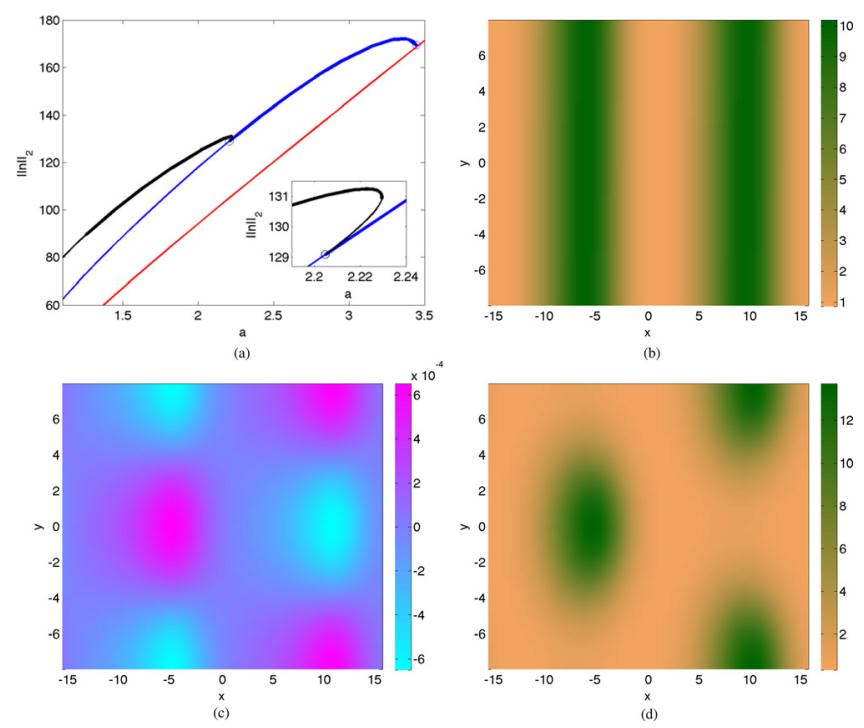

FIG. 6. Analogue of Figure 5 for stripe-rhomb bifurcation for $2 c=182.5$ under cylinder geometry. Periodic extension in both dimensions of (c) and (d) yields a rhombic pattern. Here, (d) is the solution on the black branch in (a) at the instability $a \approx 1.2$. so the stripe-rhomb bifurcation is only slightly delayed because it does not immediately satisfy the boundary conditions. In Figure 6, the striped pattern does remain stable up to the bifurcation shown.

The stripe-rectangle and stripe-rhomb bifurcations are found to be always subcritical, but relatively quickly the branch folds back, so that it appears supercritical on the larger parameter scale. The methods are not restricted to $\kappa=k_{T}$; the computations presented in Figure 7 show a rhombic pattern with both acute and obtuse angles for long $x$-wavelengths, which occur for larger $c$-values.

\section{B. No advection: Transverse instability of long wavelength striped patterns}

In this mathematically more technical section, we consider long wavelength striped patterns of the extended Klausmeier model (1) in absence of advection $(c=0)$ and establish instability with respect to perturbations along the stripes $(\ell>0)$ in the spirit of results on solitary homoclinic stripes. ${ }^{19,32,35}$

By scaling (1) into the form of the Gray-Scott model, we may use results that have already been derived for this model. Without advection and in a single space dimension, (1) is given by

$$
\begin{aligned}
w_{t} & =d w_{x x}+a-w-w n^{2}, \\
n_{t} & =n_{x x}-m n+w n^{2},
\end{aligned}
$$

and can be scaled into the standard form of the Gray-Scott equation,

$$
\begin{aligned}
& u_{T}=u_{X X}+A(1-u)-u v^{2}, \\
& n_{T}=D v_{X X}-B v+u v^{2},
\end{aligned}
$$

by setting

$$
u(X, T)=\frac{1}{a} w(x, t), v(X, T)=\frac{1}{a} n(x, t),
$$

with
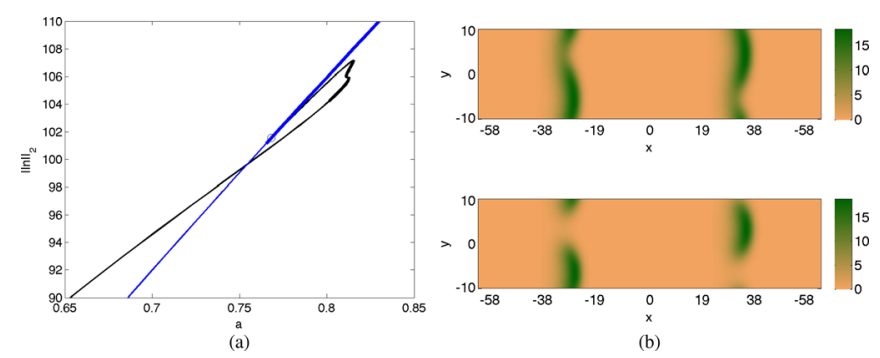

FIG. 7. (a) Stripe-rhomb bifurcation diagram of (1) for $2 c=365$ ( $d=500$, $m=0.45$ ) on a domain with periodic boundary conditions: striped patterns with wavenumber $\kappa=0.1$ (blue), stripe-rhomb patterns (black). Note that most of the solutions on the rhomb branch are unstable (even on this small domain), as their locus is plotted with a thin line. (b) Rhomb patterns (when periodically extended in both directions); upper panel: at the stability change for $a \approx 0.8$ on the lower part of the black branch, lower panel: in the unstable regime for $a \approx 0.65$. 


$$
T=a^{2} t, \quad X=\frac{a}{\sqrt{d}} x
$$

so that

$$
A=\frac{1}{a^{2}}, \quad B=\frac{m}{a^{2}}, \quad D=\frac{1}{d} .
$$

The dynamics of the Gray-Scott model are largely determined by the interplay between two small parameters. $^{11,12,33,34}$ Following Refs. 11 and 12, we therefore introduce

$$
U(\xi, \tau)=\frac{\sqrt{A}}{B \sqrt{B D}} u(X, T), \quad V(\xi, \tau)=\frac{\sqrt{B D}}{\sqrt{A}} v(X, T),
$$

with

$$
\tau=B T, \quad \xi=\frac{\sqrt{B}}{\sqrt{D}} X
$$

which transforms (39) into

$$
\begin{aligned}
D U_{\tau} & =U_{\xi \xi}-\epsilon^{2}\left[U V^{2}-\epsilon \delta\left(1-\frac{\delta}{\epsilon} U\right)\right], \\
V_{\tau} & =V_{\xi \xi}+U V^{2}-V,
\end{aligned}
$$

with

$$
\epsilon=\frac{\sqrt{A}}{B}, \delta=\sqrt{B D}
$$

An existence result on patterns $\left(U_{\mu}(\xi), V_{\mu}(\xi)\right)$ with a long wavelength $\mathcal{T}(\mu)$, with $\mu>1$ an amplitude parameter chosen for the parametrization, based on literature on the Gray-Scott model, is presented in Appendix C (Theorem 4). Here, geometric singular perturbation theory is used; the small parameter exploited is given by $\epsilon=a / m$. Note that in the long wavelength limit considered here, the $V_{\mu}(\xi)$ - component associated to plant biomass is strongly localized, while $W_{\mu}(\xi)$ varies on a larger scale. The existence of $1 \mathrm{D}$ patterns is equivalent to the existence of striped patterns in $2 \mathrm{D}$. Below, $\eta$ is a scaled version of the second spatial dimension $y$ the same way as $\xi$ relates to $x$, see (42).

To investigate the spectral stability of the striped pattern $\left(U_{\mu}(\xi), V_{\mu}(\xi)\right)$ on the full plane, so $(\xi, \eta) \in \mathbb{R}^{2}$, we set

$$
\begin{aligned}
& (U(\xi, \eta, \tau), V(\xi, \eta, \tau)) \\
& \quad=\left(U_{\mu}(\xi)+u(\xi) e^{\mathrm{i} \ell \eta+\lambda \tau}, V_{\mu}(\xi)+v(\xi) e^{\mathrm{i} \ell \eta+\lambda \tau}\right),
\end{aligned}
$$

with $\ell \in \mathbb{R}$ and $\lambda=\lambda(\mu, \ell) \in \mathbb{C}$. The linearized stability problem for (43) reads

$$
\begin{aligned}
D \lambda u & =u_{\xi \xi}-\ell^{2} u-\varepsilon^{2}\left[V_{\mu}^{2} u+2 U_{\mu} V_{\mu} v-\delta^{2} u\right], \\
\lambda v & =v_{\xi \xi}-\ell^{2} v+V_{\mu}^{2} u+2 U_{\mu} V_{\mu} v-v .
\end{aligned}
$$

We introduce $\hat{\ell}$ by

$$
\ell=\sqrt{D} \hat{\ell}
$$

and write (45) as a coupled system of Sturm-Liouville-type equations

$$
\begin{gathered}
u_{\xi \xi}-D\left[\hat{\ell}^{2}+\lambda-\frac{\varepsilon^{2} \delta^{2}}{D}\right] u=\varepsilon^{2}\left[V_{\mu}^{2} u+2 U_{\mu} V_{\mu} v\right], \\
v_{\xi \xi}+\left[2 U_{\mu} V_{\mu}-\left(1+\lambda+D \hat{\ell}^{2}\right)\right] v=-V_{\mu}^{2} u .
\end{gathered}
$$

This system can equivalently be written as a fourdimensional linear system for $\phi(\xi)=(u(\xi), p(\xi), v(\xi), q(\xi))$ with $p=\frac{1}{\varepsilon} \dot{u}$ and $q=\dot{v}$,

$$
\dot{\phi}=\mathcal{A}_{\mu}(\xi ; \lambda, \hat{\ell}) \phi
$$

where the dot denotes differentiation with respect to $\xi$ and $\mathcal{A}_{\mu}(\xi ; \lambda, \hat{\ell})$ is a $\xi$-periodic matrix,

$$
\mathcal{A}_{\mu}(\xi ; \lambda, \hat{\ell})=\left(\begin{array}{cccc}
0 & \varepsilon & 0 & 0 \\
\varepsilon\left[V_{\mu}^{2}(\xi)+\frac{D}{\varepsilon^{2}}\left(\hat{\ell}^{2}+\lambda\right)-\delta^{2}\right] & 0 & 2 \varepsilon U_{\mu}(\xi) V_{\mu}(\xi) & 0 \\
0 & 0 & 0 & 1 \\
-V_{\mu}^{2}(\xi) & 0 & -2 U_{\mu}(\xi) V_{\mu}(\xi)+\left(1+\lambda+D \hat{\ell}^{2}\right) & 0
\end{array}\right),
$$

The stability problem (47) that is equivalent to (48) has with period $\mathcal{T}(\mu)$ (see Theorem 4, Appendix C). For any $\mu>1$ and $\hat{\ell} \in \mathbb{R}$, system (48) determines a spectral problem for $\lambda=\lambda_{\mu}(\hat{\ell}) \in \mathbb{C}$ (e.g., in the space of complex-valued bounded uniformly continuous functions $B U C\left(\mathbb{R}^{2}, \mathbb{C}^{4}\right)$ ).

Following Ref. 23, and in the approach of Ref. 59, similar to (38), the eigenvalue problem (48) is considered on the fundamental interval $\left[-\frac{1}{2} \mathcal{T}(\mu), \frac{1}{2} \mathcal{T}(\mu)\right]$ with $\gamma$-twisted periodic boundary condition,

$$
\phi\left(\frac{1}{2} \mathcal{T}(\mu)\right)=\gamma \phi\left(-\frac{1}{2} \mathcal{T}(\mu)\right)
$$

for $\gamma \in S^{1} \subset \mathbb{C}$ on the unit circle. a structure that is very similar to that of the existence problem. In fact, it can be shown by directly applying the approach of Refs. 14 and 59 that the $v$-component of $\phi$ is strongly localized and exponentially small outside a fast interval $\mathcal{I}_{f}$, completely similar to $V_{\mu}(\xi)$ (Theorem 4). As a consequence, the slow reduced limit problem for the $u$ component of $\phi$, that is defined in the regions $\left[-\frac{1}{2} \mathcal{T}(\mu), \frac{1}{2} \mathcal{T}(\mu)\right] \mathcal{I}_{f}$, is given by

$$
u_{\xi \xi}-D\left[\hat{\ell}^{2}+\lambda-\frac{\epsilon^{2} \delta^{2}}{D}\right] u=0,
$$


up to exponentially small corrections. Hence, outside $\mathcal{I}_{f}, u(\xi)$ is given by a combination of exponential functions in the spatial variable $\sqrt{D} \xi$, under the assumption that $\frac{\varepsilon^{2} \delta^{2}}{D}$ is small enough, or more formally that $\varepsilon \delta \ll \sqrt{D}$. However, the length of the fundamental interval $\left[-\frac{1}{2} \mathcal{T}(\mu), \frac{1}{2} \mathcal{T}(\mu)\right]$ is of order $\frac{1}{\varepsilon \delta}$ (see (C1)): if $\varepsilon \delta \ll \sqrt{D}$, then an asymptotically bounded solution of (51) will be exponentially small at the boundaries of $\left[-\frac{1}{2} \mathcal{T}(\mu), \frac{1}{2} \mathcal{T}(\mu)\right]$. As a consequence, the entire family $\lambda(\gamma), \gamma \in S^{1}$ of $\gamma$-eigenvalues will be asymptotically close to the positive eigenvalue $\lambda$ that one can obtain (at leading order) by just considering solutions of (51) that decay exponentially as $\xi \gg \frac{1}{\sqrt{D}}$. Since the rigorous validation of this statement requires an extensive analysis along the lines of Ref. 59 , we refrain from going further into the details here.

Theorem 3. Assume that the assumptions formulated in Theorem 4 hold and consider a spatially periodic striped pattern $\left(U_{\mu}(\xi), V_{\mu}(\xi)\right)$ as given by Theorem 4 . There are constants $D_{0,1}, D_{0,2}>0$, and $0<\hat{\ell}_{1}<\hat{\ell}_{2}$, such that for all $0<D<D_{0,1}, 0<\varepsilon \delta<D_{0,2} \sqrt{D}$, and $\mu \geq 1$, eigenvalue problem (48) has a family of $\gamma$-eigenvalues, $\gamma \in S^{1}$, exponentially close to a critical eigenvalue $\lambda_{\text {pole }}(\mu, \hat{\ell})$ that is at leading order given by

$$
\lambda_{\text {pole }}(\mu, \hat{\ell})=\frac{5}{4} \text { for all } \hat{\ell} \in\left(\hat{\ell}_{1}, \hat{\ell}_{2}\right) .
$$

The lengthy proof of Theorem 3 is given in Appendix D. Note that the extension of $\left(U_{\mu}(\xi), V_{\mu}(\xi)\right)$ in the $\eta$-direction is crucial for this instability result: for certain parameter combinations, one-dimensional spatially periodic patterns $\left(U_{\mu}(\xi)\right.$, $\left.V_{\mu}(\xi)\right)$ can certainly be stable. ${ }^{13}$ In these cases, $\lambda_{\text {pole }}(\mu, \hat{\ell})$ typically merges with another eigenvalue as $\hat{\ell}>0$ decreases and forms a pair of complex conjugate eigenvalues that cross through the imaginary axis as $\hat{\ell}$ decreases further, see Ref. 35 for a much more detailed analysis of the spectral curves $\lambda(1, \ell)$ associated to the stability of a homoclinic stripe, i.e., the limit $\mu \downarrow 1$.

The instability result Theorem 3 establishes that all spatially periodic striped patterns in a certain region of the 1D Busse balloon near $\kappa=0$ are unstable with respect to transverse perturbations that are spatially periodic in the $y$-direction (and provides an asymptotic approximation of the destabilizing wavenumbers). This is presented in Corollary 2.

Corollary 2. There are constants $D_{0,1}, D_{0,2}>0$ such that for all $0<D<D_{0,1}, 0<\epsilon \delta<\mathcal{D}_{0,2} \sqrt{D}$ striped patterns $\left(U_{\mu}(\xi), V_{\mu}(\xi)\right)$ as established by Theorem 4, either as solutions of (39) or (1), are spectrally unstable.

This result holds for $c=0$, but by continuity of the spectrum, the same holds for $c$ close to 0 . In the case $c \neq 0$ without reflection symmetry, the existence of spatially periodic stripes does not directly follow from the literature and requires a new approach. Both this issue and the associated stability question are considered in Ref. 50. The instability result Theorem 3 will be influenced by the advection term $c$ : we will see in Sec. IIIC that for large $c, 2 \mathrm{D}$ stable long wavelength striped patterns for (1) are found numerically.

\section{Stability of striped patterns}

We first briefly explain how numerical continuation is implemented to trace marginal stability of striped patterns of the extended Klausmeier model (1) against the various destabilization mechanisms. We recall that in 1D, perturbations about a periodic solution are represented by functions with a $\gamma$-twisted periodicity property, where $\gamma \in S^{1} \subset \mathbb{C}$ is on the unit circle.

By translation invariance of (1), for $\gamma=1$, there is always a neutrally stable eigenvalue $\lambda(1)=0$. Since $\operatorname{Re}(\lambda(\gamma))$ is invariant with respect to complex conjugation of $\gamma$, this leads to genericity of an instability where the curve of $\operatorname{Re}(\lambda)$ at $\lambda=0$ changes from concave to convex. This destabilization mechanism is known as the Eckhaus or sideband instability, which is known to be the primary destabilization mechanism near supercritical Turing(-Hopf) bifurcations.

The sideband instability can be traced numerically using numerical continuation ${ }^{10}$ by implementing the constraint $\frac{\partial^{2}}{\partial \gamma^{2}} \operatorname{Re}(\lambda(\gamma))=0$ at $\gamma=1 .{ }^{43,60}$ It has been found that the sideband instability forms the stability boundary far beyond onset of the Turing(-Hopf) instability. ${ }^{53,60}$

The continuation of breakup instabilities of striped patterns against perturbations with $\gamma \in S^{1}$ and $\ell \in \mathbb{R}$ can be similarly implemented by imposing constraints on $\lambda(\gamma, \ell)$. That is, $\operatorname{Re}(\lambda(\gamma, \ell))=0$ and $\frac{\partial}{\partial \ell} \operatorname{Re}(\lambda(\gamma, \ell))=0$, where $\ell$ is variable and $\gamma=1$ (stripe-rectangle breakup) or $\gamma=-1$ (stripe-rhomb breakup). Here, this is done for $2 c=0,182.5$, 365,500 , and 1000 (with $d=500, m=0.45$ ) to study the dependence of striped pattern stability on the advection $c$.

In addition, simulations with a slowly decreasing $a$ are done for $2 c=0,182.5,365,500$ (again with $d=500$, $m=0.45$ ) and a comparison is made. The small growth rates associated with the sideband instability can cause a significant delay in its onset. ${ }^{53}$ Unpredictability in the outcome of the sideband instability stems from the fact that the growth rate of the perturbations that are among the first to destabilize remain small after destabilization compared to perturbations that destabilize later. In Ref. 53, it has been shown via simulations that for (1) in 1D with a slowly changing parameter, pattern adaptation depends on the rate of change and the application of noise. In this article, we fix the rate of change to $d a / d t=-10^{-5}$ and apply no noise. The simulations are done on a $250 \times 250$ square domain with periodic boundary conditions.

In Figure 8, the Busse balloon of stable striped patterns of (1) is plotted together with the sideband and transverse (breakup) instability curves, for $c=0$ and $2 c=182.5$. Frame (a) is a more detailed version of Figure 1. The representation of a pattern by a wavenumber is not guaranteed to be $1: 1$. On the contrary, for $2 c=182.5$, a brown fold curve emerges from the lower red small amplitude curve, so that between this red curve and the fold curve, a wavenumber corresponds to two patterns. But the solutions beyond the fold are all unstable, so that on the level of the Busse balloon of stable patterns, the representation is $1: 1$. As soon as a curve crosses the fold, the plotting style in Figures 8, 12, and 13 changes to dashed to indicate that it has become less relevant.

For the case $2 c=182.5$, the primary destabilization mode for breakup changes from stripe-rhomb $(\gamma=-1)$ to stripe-rectangle $(\gamma=1)$ at $a \approx 1.96$ (and back again near $a=2.9$ ). A detailed study at $a \approx 1.96$ shows that $\gamma \neq \pm 1$ does not become the primary destabilization mechanism, see 


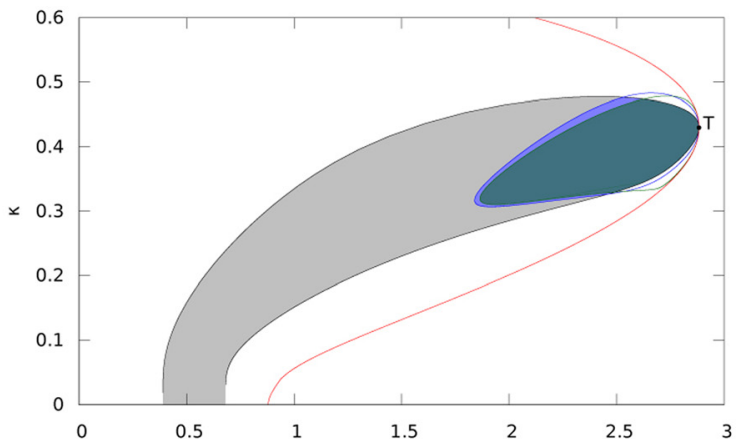

(a)

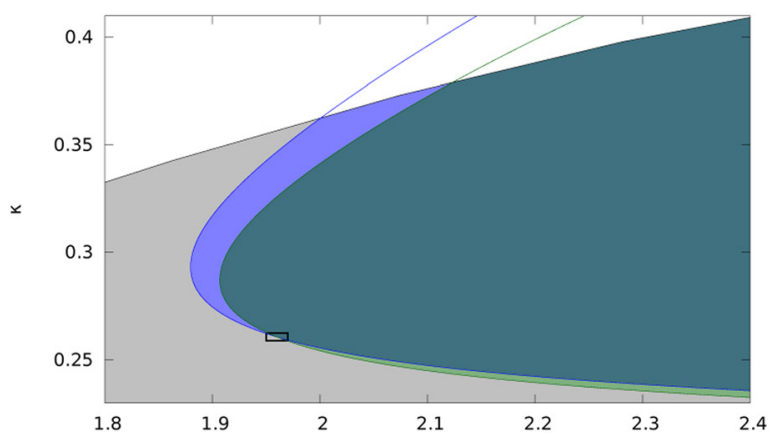

(c)

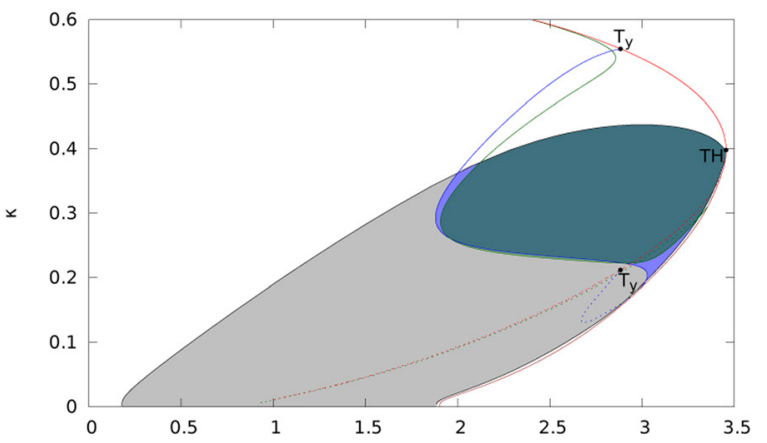

(b)

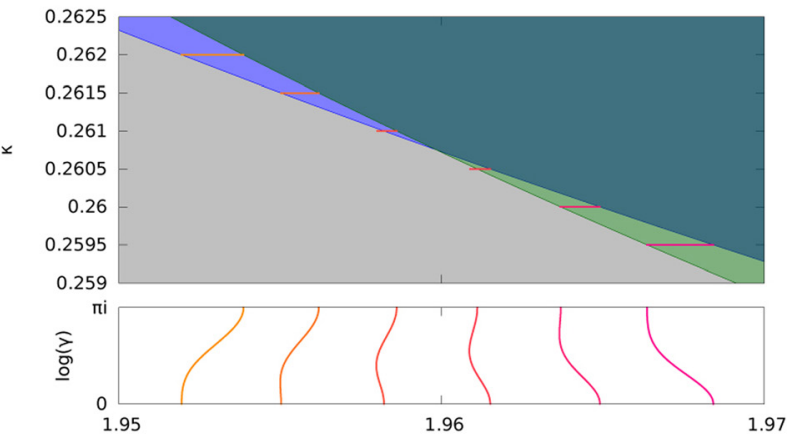

(d)

FIG. 8. Stability of striped patterns of (1) with transverse destabilization mechanisms for $d=500, m=0.45$, and (a) $c=0$ and (b) $2 c=182.5$, with Turing(-Hopf) bifurcation indicated by $T(H)$. The union of all colored regions bounded by the black sideband curve represents striped patterns that are 1D stable. The blue and green curves indicate marginal stability against stripe-rectangle and stripe-rhomb breakup, respectively. Points that represent 1D stable patterns that are stable w.r.t. striperectangle or stripe-rhomb breakup are colored blue resp. green. Full 2D stability is indicated by the dark-green (teal) combination of these colorings. Both ends of the blue curve connect to the anchor point $T_{y}\left(T=T_{y}\right.$ for $c=0$ ), see Sec. II B. (c) Part of the Busse balloon for $2 c=182.5$ showing that at $a \approx 1.96$ the stripe-rectangle and the stripe-rhomb instabilities interchange roles as primary destabilization mechanism. (d) Upper panel: magnification of region in (c) marked by the black rectangle. For selected values of $\kappa$, line segments show range of values of $a$ at marginal stability for $\log (\gamma) \in[0, \pi \mathrm{i}]$. Lower panel: $\gamma$-dependence of the line segments in the upper panel; values of $\gamma$ other then $\gamma= \pm 1$ do not act as primary destabilization mechanism.

Figure 8(d). Checks elsewhere led to the same conclusion, which is the basis for tracing breakup only for $\gamma= \pm 1$.

For $c=0$, striped patterns are seen in the simulation, but a single orientation is not always attained. In Figure 9, we show a simulation where this eventually is the case, because only then the breakup curves give a prediction for destabilization. In this case, the stripes are expected to break up before hitting the sideband curve, see Figure 8(a).

When sideband and breakup curves get close, as is the case for the transition shown in Figure 10, interaction between the destabilization mechanisms is possible. As mentioned above, the transition from one striped pattern to another through the sideband mechanism may suffer a significant delay. The modulations that arise from the sideband instability may trigger breakup before the stripe-to-stripe pattern transition has occurred. ${ }^{41}$ On the other hand, even after the breakup of a striped pattern, the system may still return to striped patterns later on, as illustrated by Figure 10 . We note that the apparent instability of the rhombic pattern of spots in Figure 10(b) does not contradict the stability that was indicated in Figure 6, since there only perturbations that fit the small domain are included.

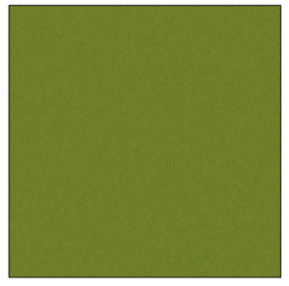

(a) $a=2.9$

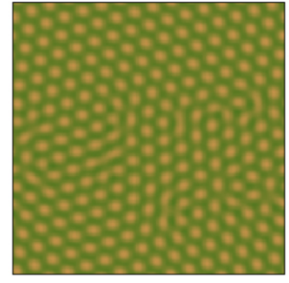

(b) $a=2.8$

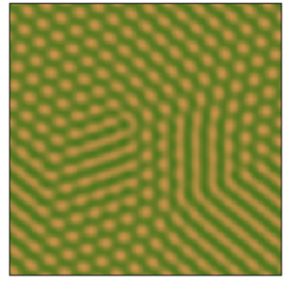

(c) $a=2.75$

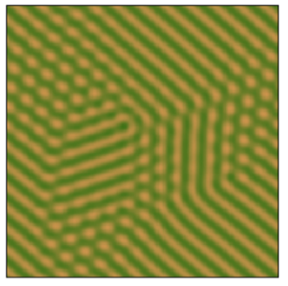

(d) $a=2.74$

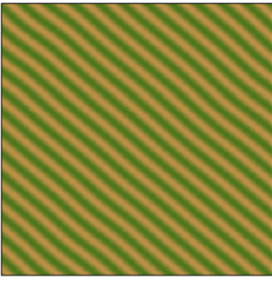

(e) $a=2.69$

FIG. 9. Frames from the simulation of (1) with slowly decreasing parameter $a, d a / d t=-10^{-5}$, for $c=0$ (with $d=500$ and $m=0.45$ ), gradient ranging from $n=0$ (sandy-brown) to $n=9.4$ (dark-green). (a) Initial condition, before Turing. (b) Pattern of gaps. (c) and (d) Gaps connect to form bare stripes, but orientation is space dependent. (e) Globally oriented striped pattern. 


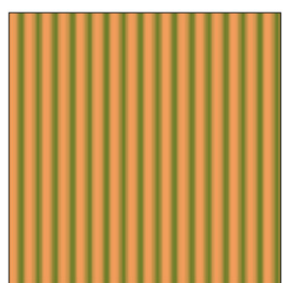

(a) $a=2.15$

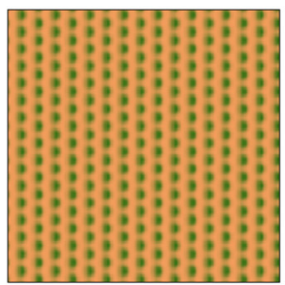

(b) $a=2.14$

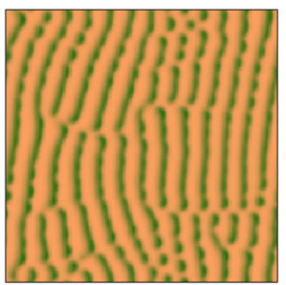

(c) $a=2.1$

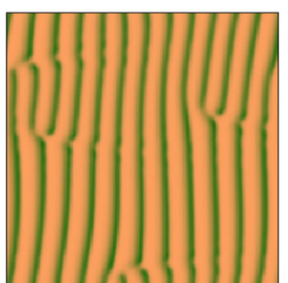

(d) $a=2.09$

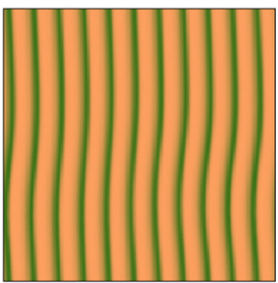

(e) $a=2.06$

FIG. 10. Frames from the simulation of (1) with slowly decreasing parameter $a$, $d a / d t=-10^{-5}$, for $2 c=182.5$ (with $d=500$ and $m=0.45$ ), gradient ranging from $n=0$ (sandy-brown) to $n=17.8$ (dark-green). Striped pattern (a) breaks up into rhombs (b), but the spots reconnect (c) and form a striped pattern with defects (d) that disappear (e).

For $2 c>0$, striped patterns aligning perpendicular to the advection start to form just below the Turing-Hopf instability. During the decrease of $a$, the system may encounter a sideband a few times first, before transverse instabilities take hold (Figure 11).

When, during a simulation, the system resides in a striped pattern, it can be represented by a single wavenumber. The plots of the wavenumbers are compared with knowledge of striped pattern stability for each of the values $2 c=0,182.5,365$, and 500 in Figure 12. The striped pattern destabilizations as observed in the simulations are in good agreement with the continuation results.
We see that in 2D, Hopf-type destabilization mechanisms that become primary destabilization mechanism ${ }^{18}$ in 1D for $\kappa \rightarrow 0$ are not relevant as long as $c$ becomes not too large. Figure 12(d) shows that around $2 c=500$, the first long wavelength (small $\kappa$ ) striped patterns become 2D stable. By increasing $c$ more and more, the transverse instabilities can be suppressed and the stability of striped patterns seems to reduce to the 1D stability of patterns, see Figure 13 for the case $2 c=1000$. Simulations for such large values of $c$ seem to require more sophisticated numerical techniques such as an operator splitting approach. ${ }^{65}$

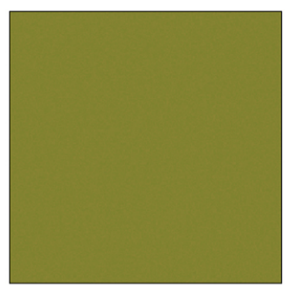

(a) $a=4.5$

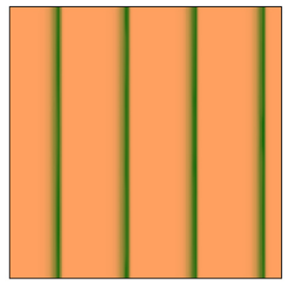

(f) $a=1$

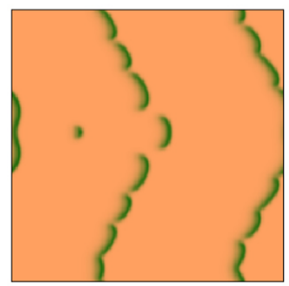

(k) $a=0.55$

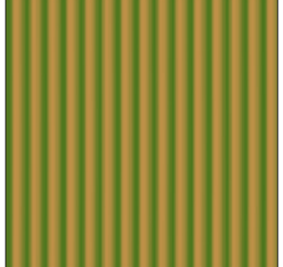

(b) $a=4.4$

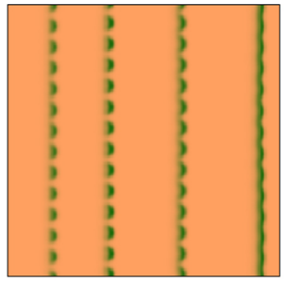

(g) $a=0.72$

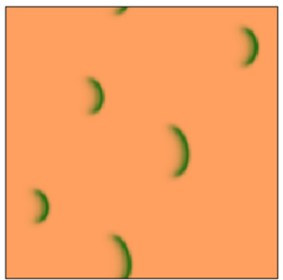

(1) $a=0.3$

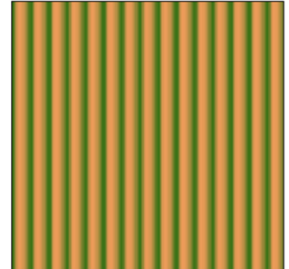

(c) $a=3.5$

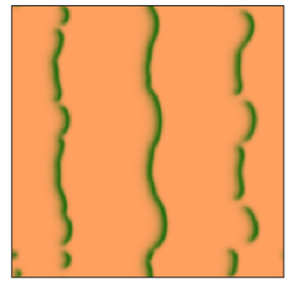

(h) $a=0.71$

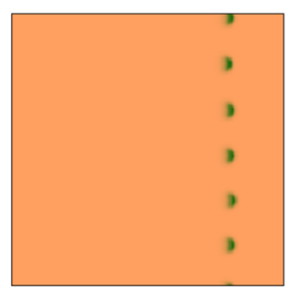

(m) $a=0.18$

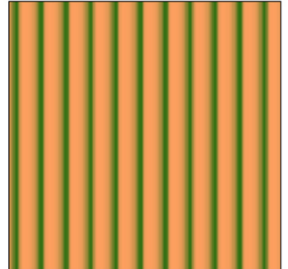

(d) $a=2.5$

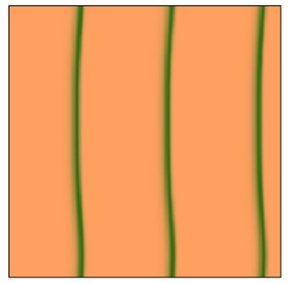

(i) $a=0.7$

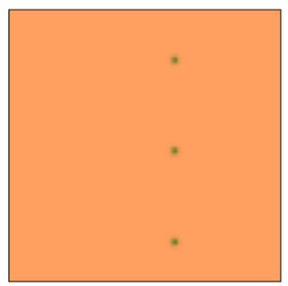

(n) $a=0.13$

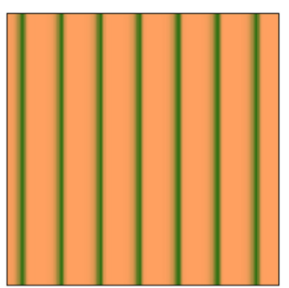

(e) $a=1.5$

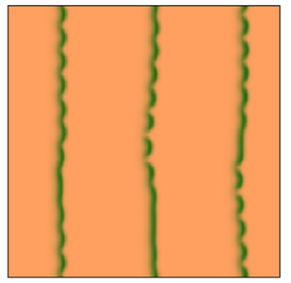

(j) $a=0.65$

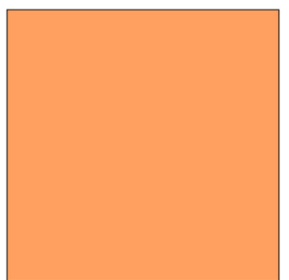

(o) $a=0.12$

FIG. 11. Frames from the simulation of (1) with slowly decreasing parameter $a, d a / d t=-10^{-5}$, for $2 c=365$ (with $d=500$ and $m=0.45$ ), gradient ranging from $n=0$ (sandy-brown) to $n=21.45$ (dark-green). Initial condition just before the Turing-Hopf bifurcation (a) and striped pattern right after (b). (c) and (f) Consecutive striped patterns after destabilization by sideband. (g)-(i) Breakup, transient dynamics and return to striped pattern. (j)-(m) Breakup, dynamics in 2D, return to dashed stripe. (n) Transverse spatial period doubling. (o) Bare desert state. 


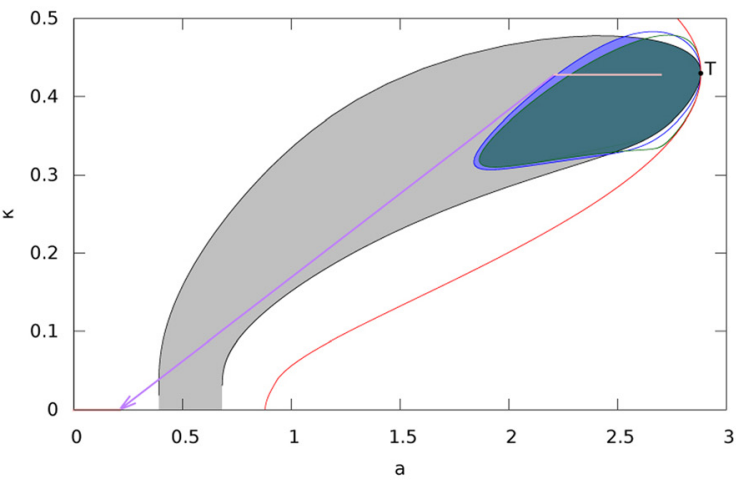

(a) $c=0$

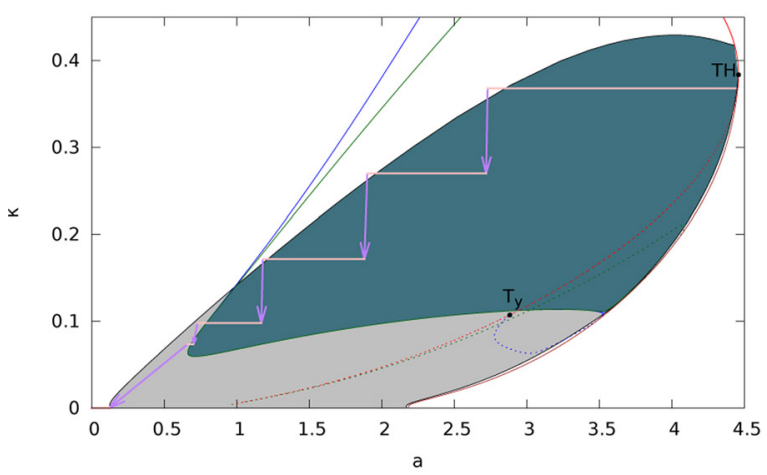

(c) $2 c=365$

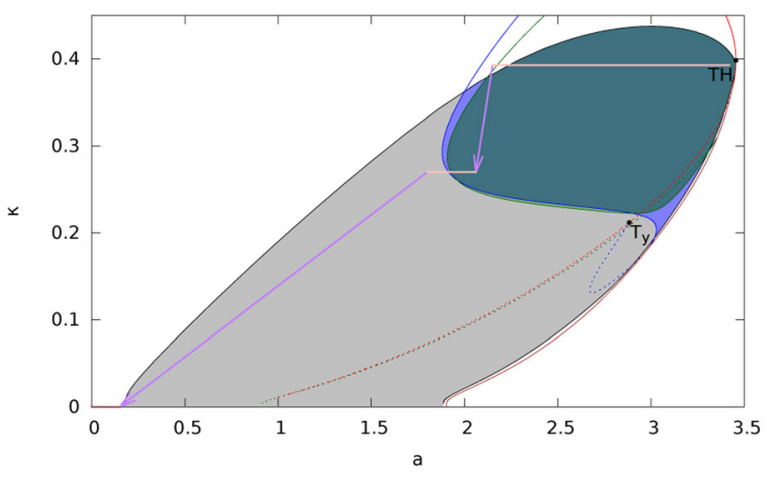

(b) $2 c=182.5$

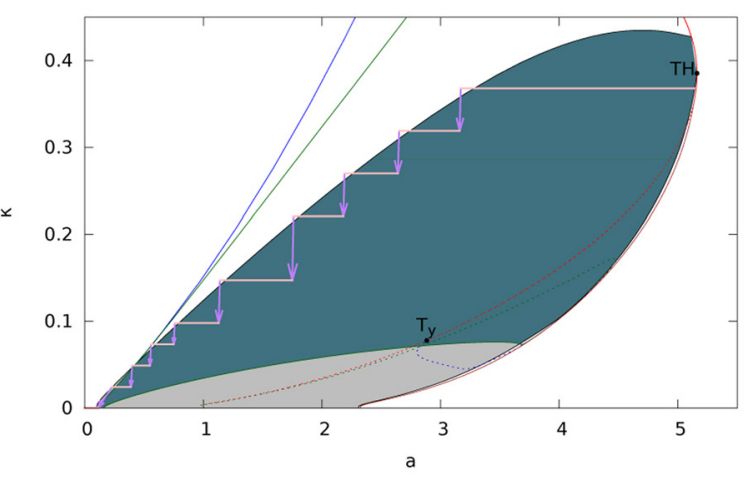

(d) $2 c=500$

FIG. 12. Stability of striped patterns of (1), see Figures 8(a) and 8(b) for the meaning of the various colored curves and regions. Connection of the blue breakup curve to the upper anchor point $T_{y}$ occurs outside the plotting range of $\kappa$. Simulations with slowly decreasing parameter $a$ included, $d a / d t=-10^{-5}$, the initial condition is a perturbation of the homogeneous state $\left(w_{+}, n_{+}\right)$at values of $a$ just before the Turing(-Hopf) bifurcation. During the simulation, the wavenumber is indicated with pink if the system resides in a striped pattern state, purple arrows in between indicate transient dynamics or residence in $2 \mathrm{D}$ states before returning to a striped pattern (or the bare desert state $\kappa=0$ ).

The above results show that by increasing the advection $c$, a larger portion of the 1D Busse balloon becomes 2D stable. We thus observe that for fixed $\kappa$, as $c$ increases, the range in $a$ where striped patterns are stable generally increases. So, the magnitude of disturbance, measured in the amount of variance in $a$, that is allowed before a striped pattern wavenumber becomes unstable, increases as $c$ increases. This confirms the result mentioned in the introduction that the ecological resilience of banded vegetation is larger on steeper slopes.

Within the choices of $c$ made in this paper, the case studied in Figures 8(c) and 8(d) is the only instance where, within the 1D Busse balloon, stripe-rectangle destabilization occurs before

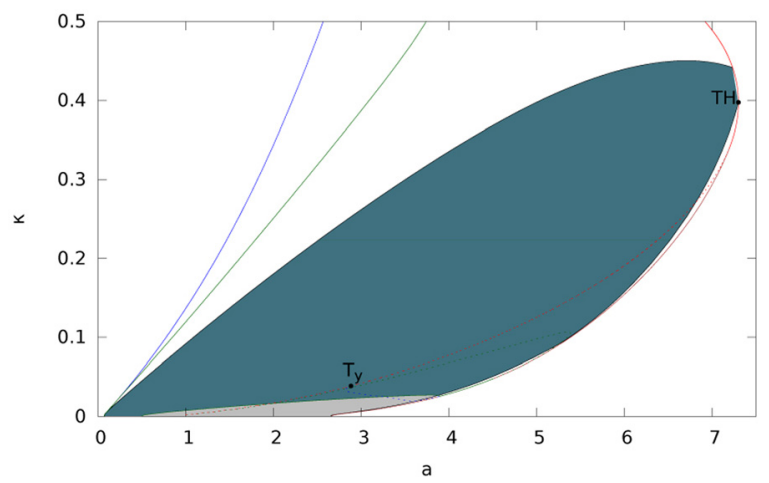

FIG. 13. Analogue of Figures $8(\mathrm{a})$ and $8(\mathrm{~b})$ for $2 c=1000$. stripe-rhomb destabilization. For larger values of $c$, represented by Figures 12(c), 12(d), and 13, the distance between the stripe-rectangle breakup and stripe-rhomb breakup curves becomes so small that they can (almost) no longer be distinguished. This can be formally understood by the observation that for large $c$, the destabilization occurs for relatively small values of the wavenumber $\kappa$. This implies that the spatially periodic patterns can be interpreted as being built from interacting localized patterns, which we expect to be of semi-strong type ${ }^{16}$ by the singularly perturbed nature of the governing equations: here, the interaction is to leading order restricted to the fastly diffusive water component. By arguments similar to those in Sec. III B, it can be expected that each family of $\gamma$-eigenvalues contracts to an asymptotically small region, so that, indeed, the $\gamma=1$ stripe-rectangle destabilization and the $\gamma=-1$ striperhomb destabilization curves become almost indistinguishable. Nevertheless, since pulses and spots in semi-strong interaction typically are repulsing, ${ }^{6,16,55}$ one also expects the rhombic patterns to be eventually the most favorable, which suggests that stripe-rhomb breakup should precede stripe-rectangle breakup, even if both curves are very close to each other.

\section{ECOLOGICAL IMPLICATIONS}

The extended Klausmeier model (1) studied in this paper is a relatively simple scaled phenomenological model; we now 
turn abstract results into qualitative predictions for arid ecosystems that could be tested empirically. The results may also help in the understanding of more complex models. In this section, we refer to striped patterns as banded vegetation. We refer to spots aligned in stripes, such as the rectangular and rhombic patterns, as dashed vegetation patterns. ${ }^{58,62}$

We recall from the introduction (result (1)) and the previous section the main numerical result: the ecological resilience of banded vegetation is larger on steep slopes, with large advection rates, than on gentle slopes. We supplement this by discussing some other implications for arid ecosystem dynamics.

Positive feedback in water-limited systems, as generated by the uptake mechanism of (1), is a key ingredient for selforganized vegetation pattern formation. ${ }^{31,44,62}$ Here, the state with uniform vegetation cover becomes unstable, because competition for water and a positive feedback between vegetation density and water harvesting capacity will create densely vegetated and more sparsely vegetated patches. Under influence of the slope, the resulting patterns are vegetation bands aligned to contours ${ }^{8,58}$ as the Turing-Hopf instability is a natural mechanism for the formation of banded vegetation perpendicular to the slope (Theorem 2).

The competition for water and the uptake mechanism continue to play an important role in pattern adaptation as environmental stress due to decreasing rainfall $a$ increases further. Competition for water between vegetation bands leads to stripe-to-stripe pattern transitions where some vegetation bands disappear and the wavenumber decreases, due to the sideband instability. ${ }^{53}$

Competition for water within each vegetation band leads to breakup by transverse instabilities. Thus, in some sense, the same mechanisms that give rise to banded vegetation patterns are eventually responsible for their breakup. The selection of a rectangular or rhombic structure at breakup depends on the interaction between vegetation bands, as sketched at the end of Sec. III C.

In Sec. II A 1, it was shown for models like (1) that vegetation bands move in uphill direction at onset. This movement is hard to establish or refute from observations, due to the small speeds that are involved. In Ref. 8, at three sites, unequivocal photographical evidence of upslope migration has been presented, but in other cases, it remains unclear. Soil characteristics not contained in (1), may be pivotal for the migration capability of vegetation patterns. ${ }^{22,47}$

In (1), we do not take into account possible mechanical action of (fast) flowing water on the strength and structure of the soil either. Particularly, in case of dashed vegetation patterns, when downslope flowpaths become long, this could be an important factor, by creating erosion (e.g., gully formation) but also possibly deposition of soil. The validity of (1) may decrease if the slope parameter $c$ increases, because these processes undermine the sheet flow of water that underpins (1). The problem of finding the correct value of $c$ can be rather complicated, as mentioned in the introduction.

From Corollary 1, we know that the Turing-Hopf bifurcation locus moves to higher rainfall $a$ as the slope $c$ increases. Since at the Turing-Hopf bifurcation banded vegetation patterns form, it would be interesting to see if observations of banded vegetation under high rainfall regimes are linked to topographies that consist of relatively steep slopes. Observations reported in Refs. 1 and 58 suggest that this may be the case.

We now turn to the numerics done in Sec. IIIC. In the simulations in the case of no slope $(c=0)$, vegetation bands still form although the orientation may (initially) be spacedependent, as in labyrinths. For gentle slopes, labyrinths are observed instead of banded vegetation. ${ }^{1,9}$ For still relatively gentle slopes $c$, i.e., $2 c \leq 182.5$ (original estimate by Ref. 31 for (1)), only a small portion of the 1D Busse balloon is $2 \mathrm{D}$ stable. In this case, no banded vegetation is expected for either small wavenumber $\kappa$ or small rainfall $a$, see Figure 8 . This is in accordance with what is reported in Ref. 9. Here at low rainfall, vegetation is mostly found not to be organized in a periodic pattern, or organized in a periodic pattern of spots. The kernel density that links to how frequent a banded vegetation pattern is observed as a function of the wavenumber $\kappa$, converges to zero well before $\kappa=0$.

In the extended Klausmeier model (1) for $d>0$, when decreasing the rainfall $a$, banded vegetation eventually breaks up into dashed patterns. This was suggested by Ref. 58 and was also found in Ref. 62. The numerics we performed show that only on very steep slopes the breakup of vegetation bands is avoided. The original Klausmeier model, where $d=0$, has been extensively studied in Ref. 52 and the references therein. Here, breakup does not occur. ${ }^{51}$ This is due to the fact that competition is now restricted within upslope segments of constant $y$, as no water is flowing in the direction of the contour. Observations of dashed patterns support incorporating a more realistic mechanism for water flow, as is done in (1). Banded vegetation breakup was not reported in Ref. 64 either.

We showed that the shape of the Busse balloon strongly depends on landscape topography. Hence, linking real vegetation patterns to desertification thresholds requires inclusion of the particular landscape setting (i.e., the slope) in which the patterns are observed.

In this paper, we refer to vegetation stripes as banded vegetation and separately identify dashed patterns. Dashed patterns are currently not treated as a separate class of patterns in observational studies and may be classified as banded vegetation instead. ${ }^{1,7,58}$ We have shown that dashed vegetation patterns naturally arise from the breakup of vegetation stripes and signify a next step in the desertification process. Therefore, a distinction between vegetation stripes and dashed patterns in the classification of vegetation patterns in observations could be considered valuable. Through observations, it may be possible to see if dashed vegetation patterns are generally found at smaller rainfall $a$ than banded vegetation. If this is the case, a restoration strategy based on dashed patterns instead of banded vegetation could in some cases be more successful, or equally successful but more economic. ${ }^{36}$ These predictions could be tested empirically.

\section{ACKNOWLEDGMENTS}

This study was supported by a grant within the Complexity program of the Netherlands Organization of 
Scientific Research (NWO). E.S. is grateful for a NWO NDNS + travel grant and thanks Universität Bremen for its kind hospitality. E.S. thanks Marco Streng and Ronald van Luijk for access to the server capella.

\section{APPENDIX A: STABILITY AGAINST LARGE WAVENUMBER PERTURBATIONS}

Lemma 4. For large $k$, solutions to the dispersion relation (6) of (8) have $\operatorname{Re}(\lambda)<0$.

Proof. In the introduction, we prescribed after (2) that $d_{1} \geq 0$ and $d_{2}>0$. If $d_{1}, d_{2}>0$, then the system (8) is characterized as being parabolic, which is well-known to imply stability against perturbations with large wavenumbers. Thus, we only need to check for the case $d_{1}=0$. We first only use that either $d_{1}=0$ or $d_{2}=0$, so that $d_{1} d_{2}=0$.

The dispersion relation (6) reads $d(\lambda, k, p, c)$ $=\lambda^{2}+\alpha_{1} \lambda+\alpha_{0}=0$, where still $\alpha_{1}=\left(d_{1}+d_{2}\right) k^{2}+O(1)$ but $\alpha_{0}=\mathrm{i} c\left(d_{1}-d_{2}\right) k^{3}+\left(c^{2}-d_{1} a_{4}-d_{2} a_{1}\right) k^{2}+O(k)$ since $d_{1} d_{2}=0$, see (9). In order to find the solution branches for large $k$, we substitute an expansion $\lambda=k^{2} \lambda_{2}+k \lambda_{1}+\lambda_{0}$ $+O(1 / k)$ with $\lambda_{j}=O(1)$.

In case $\lambda_{2} \neq 0$, we find, by comparing terms of order $k^{4}$, that $\lambda_{2}=-\left(d_{1}+d_{2}\right)<0$, which yields a parabolic asymptotically stable branch.

In case $\lambda_{2}=0$, we find the second branch (hence all solution branches of the quadratic equation). By comparing terms of order $k^{3}$, we obtain

$$
\lambda_{1}=c \frac{d_{2}-d_{1}}{d_{1}+d_{2}} \mathrm{i}
$$

which is purely imaginary. Stability is thus determined by $\lambda_{0}$, and comparing terms of order $k^{2}$ gives

$$
\lambda_{0}=\frac{\left(d_{1} a_{4}+d_{2} a_{1}\right)-c^{2}-\lambda_{1}^{2}}{d_{1}+d_{2}} .
$$

Now we use that $d_{1}=0$ so that $\lambda_{1}^{2}=-c^{2}$ and $\lambda_{0}=a_{1}$ $<0$ by the inhibitor assumption from $A 2$ in (4). So, the homogeneous steady state is stable against large wavenumber perturbations.

Note that assuming $d_{2}=0$ instead of $d_{1}=0$ in the final step of the proof would lead to $\lambda_{0}=a_{4}>0$ under assumption $A 2$ in (4). Hence, the homogeneous steady state would be unstable against "half" of the large wavenumber perturbations, and we therefore assume $d_{2}>0$.

\section{APPENDIX B: DISPERSION RELATIONS FOR STRIPED PATTERNS}

In this Appendix, we briefly outline the characterization of the spectrum of striped patterns via Floquet-Bloch decomposition. This theory may be viewed as a substitute for the Fourier transform when dealing with periodic structures. Here, it is equivalent to an Evans-function formulation using spatial dynamics, which we exploit in Sec. III B.

Let $\left(u_{*}, v_{*}\right)(t, x, y)$ denote a striped pattern of (2) that is $L$-periodic in $x$, so wavenumber $\kappa=2 \pi / L$, constant in $y$ and travels with constant speed $s$. Its spectral stability is determined by the spectrum of the linear operator arising from the linearization of (2) in a comoving frame $\xi=x-s t$ evaluated in $\left(u_{*}, v_{*}\right)$. Applying a Fourier transform in the $y$-direction with wavenumber $\ell$, we obtain the differential operator with periodic coefficients

$$
M\left(\partial_{\xi}, \xi\right)=\left(\begin{array}{cc}
d_{1}\left(\partial_{\xi}^{2}-\ell^{2}\right)+\left(c_{1}+s\right) \partial_{\xi}+a_{1}(\xi) & a_{2}(\xi) \\
a_{3}(\xi) & d_{2}\left(\partial_{\xi}^{2}-\ell^{2}\right)+\left(c_{2}+s\right) \partial_{\xi}+a_{4}(\xi)
\end{array}\right)
$$

whose spectrum is the union of spectra of the Blochoperators $M_{\mathrm{per}}(\gamma, \xi):=M\left(\partial_{\xi}-\log (\gamma) / L, \xi\right)$, with $\gamma$ on the unit circle so $\log (\gamma) \in(-\pi \mathrm{i}, \pi \mathrm{i}]$, posed on $[0,1]$ with periodic boundary conditions. ${ }^{43,48}$ Hence, the spectrum is determined by the family of eigenvalue problems $M_{\text {per }}(\gamma, \xi)-\lambda \mathrm{Id}=0$. The solutions $\lambda(\gamma)$ are referred to as $\gamma$-eigenvalues, see Sec. III B. Abstractly written, in terms of the period-map $\Phi(\lambda, \ell)$ of the evolution of this ordinary differential equation for $\gamma=1$, the expression

$$
d(\lambda, \gamma, \ell)=\operatorname{det}(\Phi(\lambda, \ell)-\gamma)
$$

is the dispersion relation analogous to the case of homogeneous steady states, which is holomorphic in $\lambda, \gamma$, and $\ell{ }^{43}$

The cases that are traced by numerical continuation in Sec. III C are $\gamma= \pm 1$, the corresponding eigenfunctions (perturbations) have distinct periodicity properties. We first restrict to $\ell=0$, so perturbations $(\tilde{u}, \tilde{v})$ that are constant in the $y$-direction. Suppose that $(\tilde{u}, \tilde{v})(\xi)$ solves $(\mathrm{B} 1)$, then

$$
\tilde{u}\left(\xi+\frac{2 \pi}{\kappa}\right)=\gamma \tilde{u}(\xi)
$$

and similarly for $\tilde{v}$.

From this, it is clear that for $\gamma=1$, the wavenumber of the perturbation is $\tilde{\kappa}=\kappa$. One of the perturbations corresponding to a solution of (B1) for $\gamma=1$ is the translation mode with eigenvalue $\lambda=0$. The solutions to (B1) consist of curves of spectrum, where $\operatorname{Re}(\lambda)$ is invariant with respect to complex conjugation of $\gamma$, which leads to genericity of the aforementioned sideband instability where the curve of $\operatorname{Re}(\lambda)$ locally changes from concave to convex at $\lambda=0$.

For $\gamma=-1$, it holds that $\tilde{\kappa}=\kappa / 2$, so the perturbation has twice the wavelength of the underlying striped pattern. This links to spatial period doubling relevant in Ref. 53.

Perturbations of striped patterns with non-trivial $y$-dependence are represented by products of a perturbation in $x$ and a perturbation in $y$, as treated in Sec. III A. 


\section{APPENDIX C: NO ADVECTION: EXISTENCE OF LONG WAVELENGTH PATTERNS}

The existence of stationary spatially periodic patterns for the scaled Gray-Scott model (43) in 1D follows directly from Ref. 13, Theorem 2.2 (which is itself based on Ref. 17, Theorem 4.2).

Theorem 4. There exist $\epsilon_{0}, \delta_{0}>0$ such that for every $0<\epsilon<\epsilon_{0}$ and $0<\delta<\epsilon \delta_{0}$, (43) has a family of stationary spatially periodic solutions $\left(U_{\mu}(\xi), V_{\mu}(\xi)\right)$, parameterized by $\mu>1$. Each periodic solution has a well-defined wavelength $\mathcal{T}(\mu)$ in the $\xi$-direction, at leading order given by

$$
\mathcal{T}(\mu)=\frac{2}{\epsilon \delta} \log \frac{\mu+1}{\sqrt{\mu^{2}-1}} .
$$

A periodic solution $\left(U_{\mu}(\xi), V_{\mu}(\xi)\right)$ can be translated (in the $\xi$-direction) in such a way that it is symmetric w.r.t. $\xi=0$ on a fundamental $\xi$-interval $\left[-\frac{1}{2} \mathcal{T}(\mu), \frac{1}{2} \mathcal{T}(\mu)\right]$. For such a solution, on a fast subinterval $\mathcal{I}_{f}=\left[-\frac{1}{\sqrt{\varepsilon}}, \frac{1}{\sqrt{\varepsilon}}\right]$ $\subset\left[-\frac{1}{2} \mathcal{T}(\mu), \frac{1}{2} \mathcal{T}(\mu)\right], U_{\mu}(\xi) \equiv 3 \mu$ is constant while $V_{\mu}(\xi)$ $=\frac{1}{2 \mu} \operatorname{sech}^{2}\left(\frac{1}{2} \xi\right)$ is the homoclinic solution of the fast reduced limit problem

$$
V_{\xi \xi}-V+3 \mu V^{2}=0,
$$

both up to corrections of $\mathcal{O}(\varepsilon)$. On the slow subintervals $\left[-\frac{1}{2} \mathcal{T}(\mu), \frac{1}{2} \mathcal{T}(\mu)\right] \mathcal{I}_{f}, V_{\mu}(\xi) \equiv 0$ up to exponentially small corrections, and $U_{\mu}(\xi)$ is at leading order given by a hyperbolic cosine solution of the slow reduced limit problem

$$
U_{\xi \xi}-\varepsilon^{2} \delta^{2} U+\varepsilon^{3} \delta=0 .
$$

Note that the earlier versions of this theorem concern special cases of the present theorem, since the choices of parameters $A, B, D$ in (39) are less general than here. ${ }^{13,17}$ This does, however, not influence the proof of the result that can be directly copied from Ref. 17. Note also that the limit $\mu \downarrow 1$, i.e., $\mathcal{T}(\mu) \rightarrow \infty$, reproduces the existence of the wellknown solitary homoclinic pulse solution of the Gray-Scott model, see Refs. 13, 17, and 33-35 and the references therein. Of course, the present result can be "translated" directly into an existence result for periodic patterns in the original model (1), under the assumption on the parameters $(a, m, d)$ of (1) that

$$
\varepsilon=\frac{a}{m}<\varepsilon_{0} \text { and } \frac{\delta}{\varepsilon}=\frac{m \sqrt{m}}{a^{2} d}<\delta_{0}
$$

and (40), (44) hold for certain $\varepsilon_{0}, \delta_{0}>0$. We refrain from giving a fully detailed rewritten version of Theorem 4 in terms of (1).

Since existence result Theorem 4 only establishes the existence of long wave length spatially periodic patterns, the results obtained in this section are only valid for wave number $\kappa$ small enough, i.e., in regions of the Busse balloon sufficiently close to the homoclinic limit $\kappa \rightarrow 0$.

The quantitative aspects of Theorem 4 may be used to analytically derive asymptotically accurate approximations of the right boundary of the Busse balloon near $\kappa=0$, see
Figure 1. This boundary has the character of a saddle-node bifurcation, and it is associated to the case in which $\varepsilon$ becomes so large that the pulse self-replication mechanism is triggered, see Refs. 13 and 60 and references therein. It is, in fact, quite surprising that the present theory appears to be valid in Figure 1, where $a \approx 0.68$ at this boundary, which implies that $\varepsilon=\frac{a}{m} \approx 1.5$ for $m=0.45$. Note that this agrees completely with the critical saddle-node/self-replication value of $\varepsilon$ as can be deduced from Ref. 13, that was obtained by careful numerical experiments on the critical magnitude of $\varepsilon$ for which the methods developed there, and used here, are valid.

\section{APPENDIX D: PROOF OF THEOREM 3}

We do not intend to present the proof of Theorem 3 (Sec. IIIB) in its full analytical detail: we will sketch the main ideas following the Evans function approach as developed in Refs. 14 and 15. To facilitate the exposition, we also impose another (formal) conditions on the relative magnitude of parameter $D$ compared to the asymptotically small parameters $\varepsilon$ and $\delta$ introduced in (44): $D \ll \epsilon^{2}$. Thus, we assume throughout this proof that

$$
\epsilon^{2} \delta^{2} \ll D \ll \epsilon^{2} \ll 1
$$

This additional condition is not essential to the validity of the instability result.

Since $V_{\mu}(\xi)$ is exponentially small outside the fast region $\mathcal{I}_{f}$ (Theorem 4), it immediately follows that the matrix $\mathcal{A}_{\mu}(\xi ; \lambda, \hat{\ell})$ is exponentially close to the constant coefficient limit

$$
\begin{aligned}
\mathcal{A}_{\mu}^{\infty}(\lambda, \hat{\ell}) & =\lim _{\xi \rightarrow \pm \infty} \mathcal{A}_{\mu}(\xi ; \lambda, \hat{\ell}) \\
& =\left(\begin{array}{cccc}
0 & \varepsilon & 0 & 0 \\
\frac{D}{\varepsilon}\left(\hat{\ell}^{2}+\lambda\right)-\varepsilon \delta^{2} & 0 & 0 & 0 \\
0 & 0 & 0 & 1 \\
0 & 0 & 1+\lambda+D \hat{\ell}^{2} & 0
\end{array}\right)
\end{aligned}
$$

outside $\mathcal{I}_{f}$. Note that in a rigorous framework, the limit $\xi$ $\rightarrow \pm \infty$ should be replaced by $\xi \rightarrow \pm \frac{1}{2} \mathcal{T}(\mu)$, which will not have a leading order effect on the outcome of the analysis (as discussed briefly in Sec. IIIB). This matrix has eigenvalues $\operatorname{Re}\left(\Lambda_{\mu, 1}(\lambda, \hat{\ell})\right)>\operatorname{Re}\left(\Lambda_{\mu, 2}(\lambda, \hat{\ell})\right)>\operatorname{Re}\left(\Lambda_{\mu, 3}(\lambda, \hat{\ell})\right)>\operatorname{Re}\left(\Lambda_{\mu, 4}(\lambda, \hat{\ell})\right)$,

$$
\begin{aligned}
\Lambda_{\mu, 1,4}(\lambda, \hat{\ell}) & = \pm \sqrt{1+\lambda+D \hat{\ell}^{2}}= \pm \sqrt{1+\lambda}+\mathcal{O}(D), \\
\Lambda_{\mu, 2,3}(\lambda, \hat{\ell}) & = \pm \sqrt{D} \sqrt{\lambda+\hat{\ell}^{2}-\frac{\varepsilon^{2} \delta^{2}}{D}} \\
& = \pm \sqrt{D} \sqrt{\lambda+\hat{\ell}^{2}}+\mathcal{O}\left(\frac{\varepsilon^{2} \delta^{2}}{D}\right)
\end{aligned}
$$

under the assumptions in (D1) and for $\hat{\ell},|\lambda|=\mathcal{O}(1)$, and associated eigenvectors 


$$
\begin{aligned}
& E_{\mu, 1,4}(\lambda, \hat{\ell})=(0,0,1, \pm \sqrt{1+\lambda}+\mathcal{O}(D)) \\
& E_{\mu, 2,3}(\lambda, \hat{\ell})=\left(1, \pm \sqrt{D} \sqrt{\lambda+\hat{\ell}^{2}}+\mathcal{O}\left(\frac{\varepsilon^{2} \delta^{2}}{D}\right), 0,0\right) .
\end{aligned}
$$

By the theory developed in Refs. 14 and 15, linear system (48) has four independent solutions $\phi_{\mu, j}(\xi ; \lambda, \hat{\ell})$, such that

$$
\begin{aligned}
& \lim _{\xi \rightarrow-\infty} \phi_{\mu, j}(\xi ; \lambda, \hat{\ell}) e^{-\Lambda_{\mu, j}(\lambda, \hat{\ell}) \xi}=E_{\mu, j}(\lambda, \hat{\ell}), \quad j=1,2, \\
& \lim _{\xi \rightarrow \infty} \phi_{\mu, j}(\xi ; \lambda, \hat{\ell}) e^{-\Lambda_{\mu, j}(\lambda, \hat{\ell}) \xi}=E_{\mu, j}(\lambda, \hat{\ell}), \quad j=3,4 .
\end{aligned}
$$

This especially implies $\lim _{\xi \rightarrow-\infty} \phi_{\mu, 1,2}(\xi ; \lambda, \hat{\ell})=(0,0$, $0,0)$ and $\lim _{\xi \rightarrow \infty} \phi_{\mu, 3,4}(\xi ; \lambda, \hat{\ell})=(0,0,0,0)$; (D5) determines $\phi_{\mu, 1,2}(\xi ; \lambda, \hat{\ell})=0$ uniquely, and since $\mathcal{A}_{\mu}(\xi ; \lambda, \hat{\ell})$ is exponentially close to $\mathcal{A}_{\mu}^{\infty}(\lambda, \hat{\ell})$, the fast transmission function $t_{\mu, f}(\lambda, \hat{\ell}): \mathbb{C} \times \mathbb{R} \rightarrow \mathbb{C}$ can be defined by

$$
\lim _{\xi \rightarrow \infty} \phi_{\mu, 1}(\xi ; \lambda, \hat{\ell}) e^{-\Lambda_{\mu, 1}(\lambda, \hat{\ell}) \xi}=t_{\mu, f}(\lambda, \hat{\ell}) E_{\mu, 1}(\lambda, \hat{\ell}),
$$

where for any given $\mu>1$ and $\hat{\ell} \in \mathbb{R}, t_{\mu, f}(\lambda, \hat{\ell})$ is analytic as a function of $\lambda .^{14,15}$

As a direct application of the methods of Refs. 14 and 15 , it can also be shown that for $\lambda, \hat{\ell}$ such that $t_{\mu, f}(\lambda, \hat{\ell}) \neq 0$, there is a uniquely determined function $\phi_{\mu, 2}(\xi ; \lambda, \hat{\ell})$ for which

$$
\lim _{\xi \rightarrow \infty} \phi_{\mu, 2}(\xi ; \lambda, \hat{\ell}) e^{-\Lambda_{\mu, 1}(\lambda, \hat{\ell}) \xi}=(0,0,0,0)
$$

i.e., there is a unique $\phi_{\mu, 2}(\xi ; \lambda, \hat{\ell})$ that does not grow with the fast rate $\Lambda_{\mu, 1}(\lambda, \hat{\ell})$ beyond the fast interval $\mathcal{I}_{f}$. As a consequence, the slow transmission function $t_{\mu, s}(\lambda, \hat{\ell})$ can be defined by

$$
\lim _{\xi \rightarrow \infty} \phi_{\mu, 2}(\xi ; \lambda, \hat{\ell}) e^{-\Lambda_{\mu, 2}(\lambda, \hat{\ell}) \xi}=t_{\mu, s}(\lambda, \hat{\ell}) E_{\mu, 2}(\lambda, \hat{\ell}),
$$

under the assumption that $t_{\mu, f}(\lambda, \hat{\ell}) \neq 0$.

For this choice of solutions $\phi_{\mu, j}(\xi ; \lambda, \hat{\ell})$ of (48), we once again follow Refs. 14 and 15 and define the Evans function $\mathcal{D}_{\mu}(\lambda, \hat{\ell})$ by

$$
\begin{gathered}
\mathcal{D}_{\mu}(\lambda, \hat{\ell})=\operatorname{det}\left[\phi_{\mu, 1}(\xi ; \lambda, \hat{\ell}), \phi_{\mu, 2}(\xi ; \lambda, \hat{\ell}),\right. \\
\left.\phi_{\mu, 3}(\xi ; \lambda, \hat{\ell}), \phi_{\mu, 4}(\xi ; \lambda, \hat{\ell})\right] .
\end{gathered}
$$

Note that this definition is only valid for $\lambda$ not in the essential spectrum associated to (48), again seen as being defined on all of $\mathbb{R}$ (instead of on $\left[-\frac{1}{2} \mathcal{T}(\mu), \frac{1}{2} \mathcal{T}(\mu)\right]$ ), so that the essential spectrum coincides with all $\lambda=\lambda(\hat{\ell}) \in \mathbb{C}$ for which $\mathcal{A}_{\mu}^{\infty}(\lambda, \hat{\ell})$ has an eigenvalue $\Lambda_{\mu, j}(\lambda, \hat{\ell}) \in \mathbb{i} \mathbb{R}$. Since the $\operatorname{trace} \operatorname{tr}\left(\mathcal{A}_{\mu}(\xi ; \lambda, \hat{\ell})\right)=0$ (see (49)), $\mathcal{D}_{\mu}(\lambda, \hat{\ell})$ does not depend on $\xi$ and $\sum_{i=j}^{4} \Lambda_{\mu, j}(\lambda, \hat{\ell}) \equiv 0$, therefore

$$
\begin{aligned}
\mathcal{D}_{\mu}(\lambda, \hat{\ell})= & \lim _{\xi \rightarrow \infty} \operatorname{det}\left[\phi_{\mu, 1}(\xi ; \lambda, \hat{\ell}), \phi_{\mu, 2}(\xi ; \lambda, \hat{\ell}),\right. \\
& \left.\phi_{\mu, 3}(\xi ; \lambda, \hat{\ell}), \phi_{\mu, 4}(\xi ; \lambda, \hat{\ell})\right] \\
= & \lim _{\xi \rightarrow \infty} \operatorname{det}\left[\phi_{\mu, 1}(\xi) e^{-\Lambda_{\mu, 1} \xi}, \phi_{\mu, 2}(\xi) e^{-\Lambda_{\mu, 2} \xi},\right. \\
& \left.\phi_{\mu, 3}(\xi) e^{-\Lambda_{\mu, 3} \xi}, \phi_{\mu, 4}(\xi) e^{-\Lambda_{\mu, 4} \xi}\right] \\
= & \operatorname{det}\left[t_{\mu, f}(\lambda, \varepsilon) E_{\mu, 1}(\lambda, \varepsilon), t_{\mu, s}(\lambda, \varepsilon) E_{\mu, 2}(\lambda, \varepsilon),\right. \\
& \left.E_{\mu, 3}(\lambda, \varepsilon), E_{\mu, 4}(\lambda, \varepsilon)\right] \\
= & 4 \sqrt{D} t_{\mu, f}(\lambda, \hat{\ell}) t_{\mu, s}(\lambda, \hat{\ell}) \sqrt{(1+\lambda)\left(\lambda+\hat{\ell}^{2}\right),}
\end{aligned}
$$

at leading order by (D3), (D4), (D5), (D6), and (D8).

For any given $\mu>1$ and $\hat{\ell} \in \mathbb{R}$, the zeroes of $\mathcal{D}_{\mu}(\lambda, \hat{\ell})$ coincide (at leading order) with the eigenvalues of (48), counting multiplicities. ${ }^{14,15}$ Moreover, under the assumptions in (D1) and for $\hat{\ell}=\mathcal{O}(1)$, it follows by Refs. 14 and 15 that the zeroes $\lambda_{\mu, f, j}$ of the fast component $t_{\mu, f}(\lambda, \hat{\ell})$ of the decomposition of the Evans function $\mathcal{D}_{\mu}(\lambda, \ell)$ (see (D10)) are at leading order given by the eigenvalues

$$
\lambda_{\mu, f, 0}=\frac{5}{4}, \lambda_{\mu, f, 1}=0, \lambda_{\mu, f, 2}=-\frac{3}{4},
$$

of the fast reduced stability problem

$$
\left(\mathcal{L}_{f}-\lambda\right) v=v_{\xi \xi}+\left[3 \operatorname{sech}^{2} \frac{1}{2} \xi-(1+\lambda)\right] v=0
$$

that can be obtained from the $v$-equation in (47) by using the leading order approximations of $U_{\mu}(x)$ and $V_{\mu}(\xi)$ in $\mathcal{I}_{f}$ (Theorem 4), and setting $u \equiv 0$, which is natural by (D6), (D4), and the fact that $\phi_{\mu, 1}(\xi ; \lambda, \hat{\ell})$ does not have any leading order slow components for $\xi<0$.

Since $t_{\mu, s}(\lambda, \hat{\ell})$ is meromorphic and has a pole at $\lambda_{\mu, f, 0}$, as we will show below, this result does not establish the instability of $\left(U_{\mu}(\xi), V_{\mu}(\xi)\right)$. In fact, this zero-pole cancellation mechanism is called "the resolution of the NLEP paradox." 14,15

Beyond the fast interval $\mathcal{I}_{f}, \phi_{\mu, 2}(\xi ; \lambda, \hat{\ell})$ is, up to exponentially small corrections, a solution of the constant coefficient problem $\dot{\phi}=\mathcal{A}_{\mu}^{\infty}(\lambda, \hat{\ell}) \phi$ that does not have a fast growing component associated to $\Lambda_{\mu, 1}(\lambda, \hat{\ell})$ and $E_{\mu, 1}(\lambda, \hat{\ell})$, see (D7). Therefore, $\phi_{\mu, 2}(\xi ; \lambda, \hat{\ell})$ is for $\xi>0$ approximated by

$$
\begin{aligned}
\phi_{\mu, 2}(\xi ; \lambda, \hat{\ell})= & t_{\mu, s}(\lambda, \hat{\ell}) e^{\Lambda_{\mu, 2}(\lambda, \hat{\ell}) \xi} E_{\mu, 2}(\lambda, \hat{\ell}) \\
& +\tilde{t}_{\mu, s}(\lambda, \hat{\ell}) e^{\Lambda_{\mu, 3}(\lambda, \hat{\ell}) \xi} E_{\mu, 3}(\lambda, \hat{\ell}),
\end{aligned}
$$

where $\tilde{t}_{\mu, s}(\lambda, \hat{\ell})$ is a second slow transmission function that measures the slow decay of $\phi_{\mu, 2}(\xi ; \lambda, \hat{\ell})$ beyond $\mathcal{I}_{f}$, see also (D8). By construction, $\phi_{\mu, 2}(\xi ; \lambda, \hat{\ell})$ is for $\xi<0$ outside $\mathcal{I}_{f}$ approximated by

$$
\phi_{\mu, 2}(\xi ; \lambda, \hat{\ell})=e^{\Lambda_{\mu, 2}(\lambda, \hat{\ell}) \xi} E_{\mu, 2}(\lambda, \hat{\ell})
$$

by (D5). 
Under the assumptions (D1), it follows by (47) that the $u$-component of a solution $\phi(\xi)$ of (48) remains constant at leading order in the fast region $\mathcal{I}_{f}$, which implies by combining (D13), (D14), and (D4) that

$$
t_{\mu, s}(\lambda, \hat{\ell})+\tilde{t}_{\mu, s}(\lambda, \hat{\ell})=1
$$

at leading order. On the other hand, a similar comparison between (D14) for $\xi<0$ and (D13) for $\xi>0$ implies that the passage of $\phi_{\mu, 2}(\xi ; \lambda, \hat{\ell})$ over $\mathcal{I}_{f}$ must have a net effect on the $p$-component $p_{\mu, 2}(\xi ; \lambda, \hat{\ell})$ of $\phi_{\mu, 2}(\xi ; \lambda, \hat{\ell})$ at leading order given by

$$
\begin{aligned}
\Delta_{s} p_{\mu, 2}= & \lim _{\xi \downarrow} \frac{1}{\sqrt{\varepsilon}} p_{\mu, 2}(\xi ; \lambda, \hat{\ell})-\lim _{\xi \uparrow \frac{1}{\sqrt{\varepsilon}}} p_{\mu, 2}(\xi ; \lambda, \hat{\ell}) \\
= & \frac{\sqrt{D}}{\varepsilon}\left(\left(t_{\mu, s}(\lambda, \hat{\ell}) \sqrt{\lambda+\hat{\ell}^{2}}-\tilde{t}_{\mu, s}(\lambda, \hat{\ell}) \sqrt{\lambda+\hat{\ell}^{2}}\right)\right. \\
& \left.-\sqrt{\lambda+\hat{\ell}^{2}}\right) \\
= & -2 \frac{\sqrt{D}}{\varepsilon}\left(1-t_{\mu, s}(\lambda, \hat{\ell})\right) \sqrt{\lambda+\hat{\ell}^{2}}
\end{aligned}
$$

by (D15).

The net effect originates from the total change over $p_{\mu, 2}(\xi ; \lambda, \hat{\ell})$ in $\mathcal{I}_{f}$, which is by (48) and the explicit approximations of Theorem 4 given by

$$
\begin{aligned}
\Delta_{f} p_{\mu, 2} & =\int_{-\frac{1}{\sqrt{\varepsilon}}}^{-\frac{1}{\sqrt{\varepsilon}}} p(\xi) d \xi \\
& =\varepsilon \int_{-\infty}^{\infty}\left(V_{\mu}^{2}(\xi) u+2 U_{\mu}(\xi) V_{\mu}(\xi) v\right) d \xi \\
& =\varepsilon \int_{-\infty}^{\infty}\left(\frac{1}{4 \mu^{2}} \operatorname{sech}^{4} \frac{1}{2} \xi+3 v_{i n, \mu}(\xi ; \lambda) \operatorname{sech}^{2} \frac{1}{2} \xi\right) d \xi,
\end{aligned}
$$

at leading order, by (D1). Here, we have used that the $u$-component of $\phi_{\mu, 2}(\xi ; \lambda, \hat{\ell})$ is at leading order constant and equal to 1 in $\mathcal{I}_{f}$ and $v_{i n, \mu}(\xi ; \lambda)$ is defined as the unique, bounded (even) solution of

$$
\begin{aligned}
& v_{\xi \xi}+\left[3 \operatorname{sech}^{2} \frac{1}{2} \xi-(1+\lambda)\right] v=-\frac{1}{4 \mu^{2}} \operatorname{sech}^{4} \frac{1}{2} \xi, \\
& \text { i.e., } \quad v_{i n, \mu}(\xi ; \lambda)=\left(\mathcal{L}_{f}-\lambda\right)^{-1}\left(-\frac{1}{4 \mu^{2}} \operatorname{sech}^{4} \frac{1}{2} \xi\right)
\end{aligned}
$$

by (D12), the leading order approximation of the fast $v$-equation of (47), that at leading order decouples from the system.

Combining (D16) and (D17) yields an explicit expression for the slow component of the decomposition of the Evans function $\mathcal{D}_{\mu}(\lambda, \hat{\ell})$ (see (D10))

$$
\begin{aligned}
t_{\mu, s}(\lambda, \hat{\ell})= & 1+\frac{\varepsilon^{2}}{2 \sqrt{D} \sqrt{\lambda+\hat{\ell}^{2}}} \\
& \times\left[\frac{2}{3 \mu^{2}}+3 \int_{-\infty}^{\infty} v_{i n, \mu}(\xi ; \lambda) \operatorname{sech}^{2} \frac{1}{2} \xi d \xi\right]
\end{aligned}
$$

at leading order. Note that it immediately follows from the definition (D19) of $v_{i n, \mu}(\xi ; \lambda)$ that $t_{\mu, s}(\lambda, \hat{\ell})$ has (simple) poles at the zeroes $\lambda_{\mu, f, 0}$ and $\lambda_{\mu, f, 2}$ of $t_{\mu, f}(\lambda, \hat{\ell})$, i.e., at the even eigenvalues
(D11) of $\mathcal{L}_{f}$. Hence, these eigenvalues do not correspond to zeroes of $\mathcal{D}_{\mu}(\lambda, \hat{\ell})$ and thus not to spectrum associated to the stability of $U_{\mu}(\xi), V_{\mu}(\xi)$. (Since the eigenfunction of (D12) associated to $\lambda_{\mu, f, 1}=0$ is odd and the right-hand side of (D18) even as function of $\xi, \lambda_{\mu, f, 1}=0$ does persist as eigenvalue of (48). $)^{14,15}$

An (eigenvalue, eigenfunction) pair of (48) is obtained by setting $t_{\mu, s}(\lambda, \hat{\ell})=0$, in which case $\phi_{\mu, 2}(\xi ; \lambda, \hat{\ell})$ decays in both limits $\xi \rightarrow \pm \infty$ (see (D13) and (D14)), i.e., by solving

$$
-2 \sqrt{\lambda+\hat{\ell}^{2}}=\frac{\varepsilon^{2}}{2 \sqrt{D}}\left[\frac{2}{3 \mu^{2}}+3 \int_{-\infty}^{\infty} v_{i n, \mu}(\xi ; \lambda) \operatorname{sech}^{2} \frac{1}{2} \xi d \xi\right] .
$$

Since the right-hand side has a simple pole near $\lambda_{\mu, f, 0}=\frac{5}{4}$, it immediately follows that there must be a solution of (D21) near $\lambda=\frac{5}{4}$ if $\frac{\varepsilon^{2}}{\sqrt{D}}$ is small enough compared to the lefthand side of (D21). Note that this expands and confirms the arguments in Ref. 13 about the instability of spatially periodic patterns by setting $\hat{\ell}=0$ (in the more special scaling there).

In the one-dimensional $\hat{\ell}=0$ setting, the patterns $\left(U_{\mu}(\xi), V_{\mu}(\xi)\right)$ may become stable as $\frac{\varepsilon^{2}}{\sqrt{D}}$ grows in magnitude, i.e., becomes $\mathcal{O}(1)$, as is shown in Ref. 13. This mechanism is counteracted in the present two-dimensional setting by the appearance of $\hat{\ell}^{2}$ in the left-hand side of (D21): if $\hat{\ell}$ is such that $\sqrt{\lambda+\hat{\ell}^{2}}$ is large enough compared to $\frac{\varepsilon^{2}}{\sqrt{D}}$, the above argument can still be applied, leading to the zeroes $\lambda_{\text {pole }}(\mu, \hat{\ell})$ as in Theorem 3 for $\hat{\ell}$ large enough and not too close to 0 , as in the statement of Theorem 3 .

In the above derivation procedure, it is assumed that $D \hat{\ell}^{2}$ is small enough: it has been neglected as a higher order effect in the reduction of the fast $v$-equation of (47) to (D18). This implies that the left-hand side of (D21) may grow to size $\frac{L}{\sqrt{D}}$ for $L$ small enough (but a priori not beyond that). Comparing this to the magnitude of the right-hand side, that is of order $\frac{\varepsilon^{2}}{\sqrt{D}}$, implies that the instability argument can be applied as long as $\varepsilon$ is small enough, which also is the assumption under which the existence of the spatially periodic stripes has been established in Theorem 4 .

\footnotetext{
${ }^{1}$ N. Barbier, P. Couteron, and V. Deblauwe, "Case study of self-organized vegetation patterning in dryland regions of central Africa," in Patterns of Land Degradation in Drylands: Understanding Self-Organised Ecogeomorphic Systems (Springer, 2013).

${ }^{2}$ F. Borgogno, P. D'Odorico, F. Laio, and L. Ridolfi, "Mathematical models of vegetation pattern formation in ecohydrology," Rev. Geophys. 47, RG1005, doi:10.1029/2007RG000256 (2009).

${ }^{3}$ B. M. Brown, M. S. P. Eastham, and K. M. Schmidt, Periodic Differential Operators (Springer, Basel, 2013).

${ }^{4}$ F. Busse, "Non-linear properties of thermal convection," Rep. Prog. Phys. 41, 1929-1967 (1978).

${ }^{5}$ J. Carballido-Landeira, P. Taboada, and A. P. Muñuzuri, "Effect of electric field on Turing patterns in a microemulsion," Soft Matter 8, 2945 (2012).

${ }^{6} \mathrm{~W}$. Chen and M. J. Ward, "The stability and dynamics of localized spot patterns in the two-dimensional Gray-Scott model," SIAM J. Appl. Dyn. Sys. 10, 582-666 (2011).

${ }^{7}$ V. Deblauwe, personal communication (2014).

${ }^{8}$ V. Deblauwe, P. Couteron, J. Bogaert, and N. Barbier, "Determinants and dynamics of banded vegetation pattern migration in arid climates," Ecol. Monogr. 82, 3-21 (2012).

${ }^{9}$ V. Deblauwe, P. Couteron, O. Lejeune, J. Bogaert, and N. Barbier, "Environmental modulation of self-organized periodic vegetation patterns in Sudan," Ecography 34, 990-1001 (2011).
} 
${ }^{10}$ E. J. Doedel, See http://cmvl.cs.concordia.ca/auto for Auto-07p: Continuation and bifurcation software for ordinary differential equations.

${ }^{11}$ A. Doelman, W. Eckhaus, and T. J. Kaper, "Slowly-modulated two-pulse solutions in the Gray-Scott model I: Asymptotic construction and stability," SIAM J Appl. Math. 61, 1080-1102 (2000).

${ }^{12}$ A. Doelman, W. Eckhaus, and T. J. Kaper, "Slowly-modulated two-pulse solutions in the Gray-Scott model II: Geometric theory, bifurcations, and splitting dynamics," SIAM J Appl. Math. 61, 2036-2062 (2001).

${ }^{13}$ A. Doelman, R. A. Gardner, and T. J. Kaper, "Stability analysis of singular patterns in the 1-D Gray-Scott model: A matched asymptotics approach," Physica D 122, 1-36 (1998).

${ }^{14}$ A. Doelman, R. A. Gardner, and T. J. Kaper, "Large stable pulse solutions in reaction-diffusion equations," Indiana Univ. Math. J. 50, 443-507 (2001).

${ }^{15}$ A. Doelman, R. A. Gardner, and T. J. Kaper, "A stability index analysis of 1-d patterns of the Gray-Scott model,” Mem. AMS 155(737), 64 (2002).

${ }^{16}$ A. Doelman and T. J. Kaper, "Semistrong pulse interactions in a class of coupled reaction-diffusion equations," SIADS 2, 53-96 (2003).

${ }^{17}$ A. Doelman, T. J. Kaper, and P. Zegeling, "Pattern formation in the onedimensional Gray-Scott model," Nonlinearity 10(2), 523-563 (1997).

${ }^{18}$ A. Doelman, J. D. M. Rademacher, and S. van der Stelt, "Hopf dances near the tips of busse balloons," Discrete Contin. Dyn. Syst. 5, 61-92 (2012).

${ }^{19}$ A. Doelman and H. van der Ploeg, "Homoclinic stripe patterns," SIAM J. Appl. Dyn. Syst. 1(1), 65-104 (2002).

${ }^{20}$ T. Dohnal, J. D. M. Rademacher, H. Uecker, and D. Wetzel, "pde2path 2.0: Multi-parameter continuation and periodic domains," in Proceedings of the 8th European Nonlinear Dynamics Conference, ENOC 2014, 2014.

${ }^{21}$ G. P. Drazin and W. H. Reid, Hydrodynamic Stability (Cambridge University Press, 1982).

${ }^{22}$ D. L. Dunkerley, "Vegetation mosaics of arid Western New South Wales, Australia: Considerations of their origin and persistence," in Patterns of Land Degradation in Drylands: Understanding Self-Organised Ecogeomorphic Systems (Springer, 2013).

${ }^{23}$ R. A. Gardner, "On the structure of the spectra of periodic travelling waves," J. Math. Pures Appl. 9, 415-439 (1993).

${ }^{24}$ M. Golubitsky, J. W. Swift, and E. Knobloch, "Symmetries and pattern selection in Rayleigh-Benard convection," Physica D 10, 249-276 (1984).

${ }^{25} \mathrm{~K}$. Gowda, H. Riecke, and M. Silber, "Transitions between patterned states in vegetation models for semiarid ecosystems," Phys. Rev. E 89, 022701 (2014).

${ }^{26} \mathrm{P}$. Gray and S. K. Scott, "Autocatalytic reactions in the isothermal, continuous stirred tank reactor: Oscillations and instabilities in the system a + 2b $\rightarrow$ 3b, b $\rightarrow$ c," Chem. Eng. Sci. 39, 1087-1097 (1984).

${ }^{27}$ L. Gunderson, "Ecological resilience-in theory and application," Annu. Rev. Ecol. Syst. 31, 425 (2000).

${ }^{28}$ C. S. Holling, "Resilience and stability of ecological systems annual review of ecology and systematics," Annu. Rev. Ecol. Syst. 4, 1-23 (1973).

${ }^{29}$ R. Hoyle, Pattern Formation: An Introduction to Methods (Cambridge University Press, Cambridge, UK, 2006).

${ }^{30}$ J. J. S. Jerome and J.-M. Chomaz, "Extended Squire's transformation and its consequences for transient growth in a confined shear flow," J. Fluid Mech. 744, 430-456 (2014).

${ }^{31}$ C. A. Klausmeier, "Regular and irregular patterns in semi-arid vegetation," Science 284, 1826-1828 (1999).

${ }^{32}$ T. Koloklonikov, W. Sun, M. J. Ward, and J. Wei, "The stability of a stripe for the Gierer-Meinhardt model and the effect of saturation," SIAM J. Appl. Dyn. Sys. 5(2), 313-363 (2006).

${ }^{33}$ T. Kolokolnikov, M. J. Ward, and J. Wei, "The existence and stability of spike equilibria in the one-dimensional Gray-Scott model: The pulsesplitting regime," Physica D 202, 258-293 (2005).

${ }^{34}$ T. Kolokolnikov, M. J. Ward, and J. Wei, "The existence and stability of spike equilibria in the one-dimensional Gray-Scott model: The low feed rate regime," Stud. Appl. Math. 115(1), 21-71 (2005).

${ }^{35}$ T. Kolokolnikov, M. J. Ward, and J. Wei, "Zigzag and breakup instabilities of stripes and rings in the two-dimensional Gray-Scott model," Stud. Appl. Math. 116(1), 35-95 (2006).

${ }^{36}$ Y. Mau, L. Haim, and E. Meron, "Reversing desertification as a spatial resonance problem,” Phys. Rev. E 91, 012903 (2015).

${ }^{37}$ J. Merkin, R. Satnoianu, and S. Scott, "The development of spatial structure in an ionic chemical system induced by applied electric fields," Dyn. Stab. Syst.: Int. J. 15(3), 209-230 (2000).

${ }^{38}$ J. H. Merkin, R. A. Satnoianu, and S. K. Scott, "Spatiotemporal chaos in a differential flow reactor," J. Chem. Soc., Faraday Trans. 94, 1211-1216 (1998).
${ }^{39}$ M. Meyries, J. D. M. Rademacher, and E. Siero, "Quasilinear parabolic reaction-diffusion systems: User's guide to well-posedness, spectra and stability of travelling waves," SIAM J. Appl. Dyn. Syst. 13, 249-275 (2014).

${ }^{40}$ J. E. Pearson, "Complex patterns in a simple system," Science 261, 189-192 (1993).

${ }^{41}$ B. Peña, C. Pérez-García, A. Sanz-Anchelergues, D. G. Míguez, and A. P. Muñuzuri, "Transverse instabilities in chemical Turing patterns of stripes," Phys. Rev. E 68, 056206 (2003).

${ }^{42}$ A. J. Perumpanani, J. A. Sherratt, and P. K. Maini, "Phase differences in reaction-diffusion-advection systems and applications to morphogenesis," IMA J. Appl. Math. 55, 19-33 (1995).

${ }^{43}$ J. D. M. Rademacher, B. Sandstede, and A. Scheel, "Computing absolute and essential spectra using continuation," Physica D 229, 166-183 (2007).

${ }^{44}$ M. Rietkerk, M. C. Boerlijst, F. van Langevelde, R. Hillerislambers, J. van de Koppel, L. Kumar, H. H. T. Prins, and A. M. de Roos, "Self-organization of vegetation in arid ecosystems," Am. Nat. 160, 524-530 (2002).

${ }^{45}$ A. B. Rovinsky and M. Menzinger, "Chemical instability induced by a differential flow," Phys. Rev. Lett. 69, 1193 (1992).

${ }^{46}$ A. B. Rovinsky and M. Menzinger, "Self-organization induced by the differential-flow of activator and inhibitor," Phys. Rev. Lett. 70, 778-781 (1993).

${ }^{47}$ P. M. Saco, G. R. Willgoose, and G. R. Hancock, "Eco-geomorphology of banded vegetation patterns in arid and semi-arid regions," Hydrol. Earth Syst. Sci. 11, 1717-1730 (2007).

${ }^{48}$ B. Sandstede, "Stability of travelling waves," in Handbook of Dynamical Systems, II (Elsevier, 2002), pp. 983-1055.

${ }^{49}$ R. A. Satnoianu, J. H. Merkin, and S. K. Scott, "Spatio-temporal structures in a differential flow reactor with cubic autocatalator kinetics," Physica D 124, 345-367 (1998).

${ }^{50}$ L. Sewalt and A. Doelman, "Spatially periodic multi-pulse patterns in a generalized Klausmeier-Gray-Scott model" (unpublished).

${ }^{51}$ J. A. Sherratt, "An analysis of vegetation stripe formation in semi-arid landscapes," J. Math. Biol. 51, 183-197 (2005).

${ }^{52}$ J. A. Sherratt, "Pattern solutions of the Klausmeier model for banded vegetation in semi-arid environments V: The transition from patterns to desert," SIAM J. Appl. Math. 73, 1347-1367 (2013).

${ }^{53}$ K. Siteur, E. Siero, M. Eppinga, J. D. M. Rademacher, A. Doelman, and M. Rietkerk, "Beyond Turing: The response of patterned ecosystems to environmental change," Ecol. Complexity 20, 81-96 (2014).

${ }^{54} \mathrm{H}$. B. Squire, "On the stability of 3D disturbances of viscous flow between parallel walls," Proc. R. Soc. London, Ser. A 142, 621-628 (1933).

${ }^{55} \mathrm{~W}$. Sun, M. J. Ward, and R. Russell, "The slow dynamics of two-spike solutions for the Gray-Scott and Gierer-Meinhardt systems: Competition and oscillatory instabilities," SIADS 4, 904-953 (2005).

${ }^{56}$ A. Toth, D. Horvath, and W. van Saarloos, "Lateral instabilities of cubic autocatalytic reaction fronts in constant electric field," J. Chem. Phys. 111, 10964-10968 (1999).

${ }^{57}$ H. Uecker, D. Wetzel, and J. D. M. Rademacher, "pde2path: A Matlab package for continuation and bifurcation in 2D elliptic systems," Numer. Math.: Theory Methods Appl. 7, 58-106 (2014).

${ }^{58} \mathrm{C}$. Valentin, J. M. d'Herbès, and J. Poesen, "Soil and water components of banded vegetation patterns," CATENA 37, 1-24 (1999).

${ }^{59} \mathrm{H}$. van der Ploeg and A. Doelman, "Stability of spatially periodic pulse patterns in a class of singularly perturbed reaction-diffusion equations," Indiana Univ. Math. J. 54, 1219-1302 (2005).

${ }^{60}$ S. van der Stelt, A. Doelman, G. Hek, and J. D. M. Rademacher, "Rise and fall of periodic patterns for a Generalized Klausmeier-Gray-Scott model," J. Nonlinear Sci. 23, 39-95 (2013).

${ }^{61}$ B. Z. Virany, A. Szommer, A. Toth, and D. Horvath, "Lateral instability controlled by constant electric field in an acid-catalyzed reaction," Phys. Chem. Chem. Phys. 6, 3396-3401 (2004).

${ }^{62}$ J. von Hardenberg, E. Meron, M. Shachak, and Y. Zarmi, "Diversity of vegetation patterns and desertification," Phys. Rev. Lett. 87, 198101 (2001).

${ }^{63}$ R.-H. Wang, Q.-X. Liu, G.-Q. Sun, Z. Jin, and J. van de Koppel, "Nonlinear dynamic and pattern bifurcations in a model for spatial patterns in young mussel beds," J. R. Soc. Interface 6, 705-718 (2009).

${ }^{64}$ H. Yizhaq, E. Gilad, and E. Meron, "Banded vegetation: Biological productivity and resilience," Phys. A 356, 139-144 (2005).

${ }^{65}$ S. Zhao, J. Ovadia, X. Liu, Y.-T. Zhang, and Q. Nie, "Operator splitting implicit integration factor methods for stiff reaction-diffusion-advection systems," J. Comput. Phys. 230(15), 5996-6009 (2011). 